\title{
A Predictive in Vitro Risk Assessment Platform for Pro-Arrhythmic Toxicity Using Human 3D Cardiac Microtissues
}

\section{Celinda Kofron}

Brown University

Tae Yun Kim

Rhode Island Hospital

Fabiola Munarin

Brown University

\section{Arvin Soepriatna}

Brown University

\section{Rajeev Kant}

Brown University

\section{Ulrike Mende}

Rhode Island Hospital

\section{Bum-Rak Choi}

Rhode Island Hospital

Kareen Coulombe ( $\sim$ Kareen_Coulombe@brown.edu )

Brown University

\section{Research Article}

Keywords: Cardiotoxicity, drugs, environmental, cardiac

Posted Date: January 19th, 2021

DOl: https://doi.org/10.21203/rs.3.rs-147721/v1

License: (a) This work is licensed under a Creative Commons Attribution 4.0 International License. Read Full License 


\section{Abstract}

Cardiotoxicity of pharmaceutical drugs, industrial chemicals, and environmental toxicants can be severe, even life threatening, which necessitates a thorough evaluation of the human response to chemical compounds. Predicting risks for arrhythmia and sudden cardiac death accurately is critical for defining safety profiles. Currently available approaches have limitations including a focus on single select ion channels, the use of non-human species in vitro and in vivo, and limited direct physiological translation. We have advanced the robustness and reproducibility of in vitro platforms for assessing pro-arrhythmic cardiotoxicity using human induced pluripotent stem cell-derived cardiomyocytes and human cardiac fibroblasts in 3-dimensional microtissues. Using automated algorithms and statistical analyses of eight comprehensive evaluation metrics of cardiac action potentials, we demonstrate that tissue-engineered human cardiac microtissues respond appropriately to physiological stimuli and effectively differentiate between high-risk and low-risk compounds exhibiting blockade of the hERG channel (E4031 and ranolazine, respectively). Further, we show that the environmental endocrine disrupting chemical bisphenol-A (BPA) causes acute and sensitive disruption of human action potentials in the nanomolar range. Thus, this novel human $3 \mathrm{D}$ in vitro pro-arrhythmic risk assessment platform addresses critical needs in cardiotoxicity testing for both environmental and pharmaceutical compounds and can be leveraged to establish safe human exposure levels.

\section{Introduction}

Cardiotoxicity in response to drugs, chemicals and other environmental toxicants entails both structural and functional changes, and potentially lethal pro-arrhythmic effects continue to be of particular concern. Notably, drug-induced QT prolongation and Torsades de Pointes (TdP), fatal arrhythmias associated with sudden cardiac death, are the single most common cause of withdrawal or restriction of the use of drugs on the market ${ }^{1-7}$ despite intense preclinical screening in silico, in vitro, and in animal models. Additionally, the World Health Organization (WHO) estimates that environmental toxicants such as pesticides and industrial chemicals may evoke up to $23 \%$ of cardiovascular diseases globally, ${ }^{8}$ but the Environmental Protection Agency (EPA) still has little effort focused on cardiotoxicity, even with the current state-of-the-art computational approaches to chemical safety evaluation of Toxicity Forecaster (ToxCast) and Toxicity Testing in the 21st Century (Tox21). The high prevalence of drug- and chemicalinduced pro-arrhythmic cardiotoxicity that can increase the risk for stroke, heart attack, heart failure, and sudden cardiac death ${ }^{9}$ point to a continued need for more reliable and robust risk assessment efforts to predict cardiotoxic effects with high sensitivity.

Existing pro-arrhythmia assays have a few key limitations. Animal models are complex and have limited predictability of human biological responses due to species-specific differences in ion channel expression that affect depolarization and repolarization kinetics of cardiac action potentials (APs) as well as differential sensitivity to pharmacological agents. ${ }^{10,11}$ Human ether-a-go-go (hERG) channel blockade in vitro and corrected QT interval (QTC) prolongation in patients ${ }^{12,13}$ have long been used as effective and 
selective 'biomarkers' in compound screening to identify pro-arrhythmic risks that cause TdP. However, it is now recognized that a 'hERG-centric' approach does not capture all drug-induced cardiac arrhythmia mechanisms ${ }^{14-18}$ and can also lead to unnecessary discontinuation of compounds from development due to false positive outcomes despite clinical utility below an arrhythmogenic dose. ${ }^{19-21}$ More recently developed in vitro cell-based models are often limited to 2D monolayers and lack sufficient myocardial cell-cell interactions, including heterotypic interactions between cardiomyocytes and cardiac fibroblasts, which modulate excitation-contraction in cardiomyocytes and thus affect the arrhythmogenic phenotype. $^{22}$

New initiatives in toxicity screening specifically call for the development and implementation of nonanimal approaches to assess potential hazards associated with acute and chronic exposures to industrial chemicals and medical products. Groups such as the Interagency Coordinating Committee on the Validation of Alternative Methods (ICCVAM) were assembled in response to new laws across the globe and point to a strong value in platforms based on a mechanistic understanding of toxicity. ${ }^{23,24}$ Continued development of new approach methodologies (NAMs) to enhance predictive capabilities for prioritization, hazard screening, and risk assessment of cardiotoxicity is thus warranted. ${ }^{25}$

The inter-agency Comprehensive In Vitro Pro-arrhythmia Assay (CiPA) initiative has identified necessary goals for advancing cardiotoxicity testing: defining drug effects on seven human cardiac currents with automated patch clamping, using in silico models to define pro-arrhythmia risk metrics, verifying effects on cardiomyocytes derived from human induced pluripotent stem cells cultured in 2-dimensional monolayers, and validating with human clinical data for in vitro to in vivo extrapolation (IVIVE). ${ }^{26,27}$ Human induced pluripotent stem cell (hiPSC)-derived cardiomyocytes (CMs) are regarded as the future of NAMs for cardiotoxicity evaluation for their appropriate physiology, including proper human ion channel expression, AP shape, and rhythmic contractions. ${ }^{28-31}$ HiPSC-CMs show great promise in detecting druginduced pro-arrhythmic effects based on electrophysiological responses. ${ }^{32}$ One complication in the field arises from cell-to-cell and batch-to-batch variability of hiPSC-CMs, which limits comparison within a narrow and defined time within a same batch. In addition, hiPSC-CM-based models built from traditional 2D culture systems do not recapitulate the cellular architecture of native tissue. Scaffold-supported and self-assembled cultures provide a 3D environment for more mature CM function, ${ }^{33,34}$ and microtissues maximize cell-to-cell connectivity while allowing for easy visual assessment of the action potential (AP) and calcium transients through fluorescent reporters. ${ }^{35,36}$

Several groups are leveraging the utility of 3D cardiac microtissues for toxicant screening, ${ }^{22}$ focusing on cell viability, ${ }^{37,38}$ gene expression, ${ }^{39}$ and mechanical outcomes such as force generation and contractility, ${ }^{22,40-44}$ but not yet on diverse pro-arrhythmic metrics such as AP, calcium transient, and conduction velocity. Some 3D platforms that focus on arrhythmia have limited throughput (e.g., confocal microscopy ${ }^{34}$ or single microelectrode recording ${ }^{42}$ ) and focus on spontaneous beating frequency, ${ }^{45}$ intracellular calcium flux recordings to infer AP changes, ${ }^{46}$ or multi-electrode array recordings of the extracellular field potentials that can have inter test-site differences ${ }^{47-51}$. Many of these studies do not 
report characterization of reproducibility in light of batch-to-batch variability that is inherent to stem maintenance, differentiation, and purification protocols. ${ }^{52,53}$ Important nuances of excitation that these approaches are not designed to capture and assess include non-uniform reduced excitation within a single microtissue; waveform changes such as upstroke, plateau level, or recovery rate of APs to predict ion channel blockade or $\mathrm{Ca}^{2+}$ handling anomalies; APD triangulation related to intra-phase prolongation; and pro-arrhythmic triggered activity classification based on mechanism such as early- (EADs) and delayed-afterdepolarizations (DADs). $.54,55$

In this study, our objective was to fill these gaps and thereby advance predictive preclinical models of human drug-induced pro-arrhythmic risk using hiPSC-CMs and human cardiac fibroblasts (hCFs) in 3D self-assembled engineered microtissues. Utilizing optical mapping of voltage sensitive dyes, an established approach to evaluate the cardiac $\mathrm{AP}^{54}{ }^{54}$ we developed an automated analysis pipeline to produce eight comprehensive evaluation metrics of the AP with reduced bias and increased throughput for risk assessment that capture pro-arrhythmic activity. To establish the fitness of this NAM to predict arrhythmogenesis, we thoroughly evaluated variability of our platform including beat-to-beat, microtissueto-microtissue, mold-to-mold, and batch-to-batch for comprehensive statistical analysis of action potentials in response to test compounds. We validated our model with known high-risk and low-risk blockers of the hERG channel. We also evaluated a compound with poorly described human arrhythmic cardiotoxicity, bisphenol A (BPA), an industrial chemical and environmental toxicant classified as an endocrine disrupting chemical. BPA was found to alter excitation with nanomolar sensitivity, showing a dose-dependent reduction in AP duration in hiPSC-CMs, suggesting that our model is sensitive, selective, and fit for the purpose of human cardiac risk assessment.

\section{Results}

\section{Development of 3D human cardiac microtissues using hiPSC-CMs and hCFs}

To develop a predictive human cardiotoxicity model to specifically address risk of cardiac arrhythmias, we adapted a scaffold-free cardiac microtissue model we previously developed with rat primary cardiac

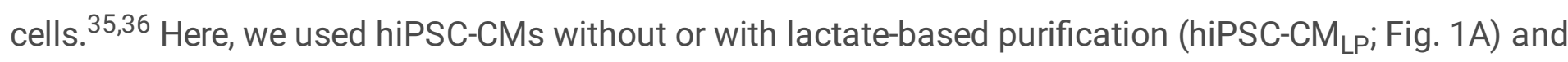
primary normal adult human cardiac fibroblasts (hCFs) to generate 35 self-assembled microtissues per non-adhesive agarose gel containing cylindrical microwells with hemispherical bottoms (Fig. 1B). The structural and functional features of these microtissues were assessed with live cell imaging (Fig. 1C), immunohistochemistry and confocal imaging (Fig. 1D), and optical mapping (Fig. 1E,F). Consistent and reliable formation of 35 viable spherical microtissues per well was achieved (Fig. 1C, Movie S1) with the addition of $5 \%$ hCF to the cultures, increasing the reliability of self-assembly to incorporate all cells in a microwell (data not shown). Microtissue size varied with plating density. For microtissues generated from lactate-purified hiPSC-CMs, a plating density of 13,500 cells per microtissue ( $95 \%$ hiPSC-CM $\mathrm{LP}_{\mathrm{LP}}$ and $5 \%$ hCF) yielded spherical microtissues $359.4 \pm 32.4 \mu \mathrm{m}$ in diameter after $1 \mathrm{~d}$ in $3 \mathrm{D}$ culture that compacted to 
$290.4 \pm 29.5 \mu \mathrm{m}$ in diameter after 5 d. A plating density of 15,000 cells per microtissue $(95 \%$ hiPSC-CM and $5 \% \mathrm{hCF}$ ) yielded spherical microtissues $400.4 \pm 24.5 \mu \mathrm{m}$ in diameter after $1 \mathrm{~d}$ and $329.4 \pm 17.5 \mu \mathrm{m}$ after $5 \mathrm{~d}$. Immunostaining with antibodies against cardiac troponin I (cTnl) and vimentin (vim) confirmed that hiPSC-CMs and hCFs self-assembled to be highly interspersed (Fig. 1D), consistent with our prior work in rat cardiac microtissues. ${ }^{35,36}$

\section{Electrophysiological characterization of 3D cardiac microtissues}

In order to evaluate excitability and sensitivity to pro-arrhythmic toxicants, APs were recorded from the human cardiac 3D microtissues with voltage sensitive dyes (Fig. 1E). Cardiac microtissues were paced at $2 \mathrm{sec}$ basic cycle length $(0.5 \mathrm{~Hz})$ and APs were recorded for 8-10 sec. Human cardiac 3D microtissues showed robust fluorescence changes tracing $\mathrm{V}_{\mathrm{m}}$ signal of APs during spontaneous and paced beats. The data analysis of APs was automated to reduce bias and achieve increased throughput (Supplemental Figure S1). Pro-arrhythmic risk metrics were defined as depicted in Fig. 1F-H: excitability, stimulation time delay between stimulation pulse and peak AP upstroke (stimulation delay time, stim $\triangle$ ), rise time of AP upstroke, AP duration (APD) to $30 \%, 50 \%$, and $80 \%$ repolarization $\left(\mathrm{APD}_{30}, \mathrm{APD}_{50}, \mathrm{APD}_{80}\right)$, AP duration to end of maximum repolarization rate $\left(A P D_{M \times R}\right.$, determined with $\left.d^{2} \mathrm{~F} / \mathrm{dt}^{2}{ }_{\mathrm{max}}\right), \mathrm{APD}$ triangulation $\left(\mathrm{APD}_{\mathrm{tri}}=\right.$ $\left.A P D_{M \times R}-A P D_{50}\right)$, and presence of EADs (\% of microtissues showing EAD).

Biological variation was low across biological replicates and experimental time (Fig. 2). Example traces show reproducibility (Fig. 2A), and an example of APD distribution across different microtissues from a single mold was normally distributed (Fig. 2B). The Kolmogorov-Smirnov test for 3 molds with a total of 105 cardiac microtissues indicated normal APD distribution $(p=0.92)$. In order to determine the statistical power of this 3D in vitro methodology, we examined the standard deviation as the variability in APD. In microtissues formed with hiPSC-CMs that did not undergo lactate purification $(70.3 \pm 3.1 \%$ cardiac troponin $T$ positive for $n=4$ batches), the mean $A_{P D}$ was $160.0 \pm 33.1 \mathrm{~ms}$ (Fig. $2 \mathrm{C}$, bottom). The standard deviation from batch to batch ("between batches") was the largest of our biological variability metrics at $>30 \mathrm{~ms}$. Microtissue-to-microtissue and mold-to-mold variability were smaller, and variability in technical replicates beat-to-beat (same microtissue) was $<10 \mathrm{~ms}$ (Fig. 2C). Action potential duration showed the expected physiological rate dependence at physiological heart rates (60-75 bpm; Fig. 2D). Power analysis of sample size suggested that $n=17$ cardiac microtissues are required to detect a $10 \%$ change in APD with $95 \%$ confidence, suggesting that testing within a single mold is sufficient for evaluating small but meaningful changes in APD measured in vitro. ${ }^{56}$ The sample size can be further reduced by using paired testing ( $\mathrm{n}=8$ based on beat-to-beat variation) on the same mold before and after toxicant exposure. In order to screen cardiotoxicity from the same microtissues before and after toxicant exposure, APDs should be stable between successive recordings. We chose a 20 min incubation period to ensure that the test compound had ample time to diffuse into the 3D cardiac microtissue, ${ }^{57}$ which is the limiting factor since typical ion channel blockers bind and act relatively quickly, in less than a minute (for example, E4031 has a binding time constant of $0.8 \mathrm{sec}^{58}$ ). Figure $2 \mathrm{E}, \mathrm{F}$ shows comparable traces and 
group data for $\mathrm{APD}_{80}$ in our model over 20 min, which enabled testing of several doses of toxicant with this acute exposure protocol on the same mold to perform paired t-tests. The addition of $5 \% \mathrm{hCF}$ to microtissues upon formation improved not only the consistency of formation and clean edges of microtissues (data not shown), but also significantly increased the number of excitable microtissues by more than $10 \%$ (Fig. $2 \mathrm{G}$ ), while slightly shortening APD by $12 \%$ (Fig. $2 \mathrm{H}$ ).

Our model was predictive of expected arrhythmia risk in pilot experiments with known drugs such as $\mathrm{I}_{\mathrm{Kr}}$ blocker E4031 (data not shown), yet the APD in these microtissues was under $200 \mathrm{~ms}$, and while within the range reported for hPSC-CMs ${ }^{59}$ this was shorter than APD typically recorded in human. ${ }^{60,61}$ To improve the reliability and robustness of our arrhythmia model, we selected for and matured our hiPSCCMs with metabolic-based lactate purification of cardiomyocytes (hiPSC-CM $\mathrm{LP}_{\mathrm{LP}}$ ). Lactate purification lengthened APD (Fig. $3 \mathrm{~A}$ ) and improved the excitability of microtissues when directly compared to experimental controls that were age-matched but not lactate purified $\left(84.4 \pm 6.5 \%\right.$ for hiPSC-CM $\mathrm{LP}_{\mathrm{LS}}$. $25.2 \pm 25.8 \%$ for hiPSC-CM) (Fig. 3B). Similar to hiPSC-CM microtissues with hCFs, the hiPSC-CM ${ }_{L P}$ microtissues with hCFs have a normal distribution (Kolmogorov-Smirnov test $p=0.580$; Fig. $3 \mathrm{C}$ ) and low variability (Fig. 3D). Batch- to-batch standard deviation was reduced by $42 \%$ compared to hiPSC-CM microtissues and beat-to-beat APD variation from the same microtissue (technical replicates) was also smaller, whereas microtissue-to-microtissue variability was similar, and mold-to-mold variability was slightly higher than variation in hiPSC-CM tissues (Fig. 3D vs. Figure $2 \mathrm{C}$ ). The cumulative probability plot for $A D_{80}$ shifted to the right with lactate purification (Fig. 3E), and APD was doubled from $130.6 \pm 54 \mathrm{~ms}$ in hiPSC-CM microtissues to $263.1 \pm 54.9 \mathrm{~ms}$ with lactate purification (Fig. 3F). All subsequent experiments were performed with microtissues generated from hiPSC-CMLP and $5 \%$ hCFs.

\section{Model Qualification: APD and arrhythmia test with known high-risk and low-risk potassium channel $\left(\mathrm{I}_{\mathrm{Kr}}\right)$ blockers}

To validate the capability of our model to predict arrhythmia risk, we exposed microtissues to the proarrhythmic hERG channel blocker E4031. APD 80 was prolonged under $2 \mu \mathrm{M}$ E4031 (Fig. 4A, B, Movies S2, S3). Cumulative distribution plots show a rightward shift with E4031 treatment (Fig. 4C). When analyzing single batches of microtissues $(n=35)$, we confirmed that $A P D_{80}$ had a normal distribution of values with $\mathrm{Q}-\mathrm{Q}$ plots (Supplemental Fig. 2). EADs were present in 54\% of microtissues with $2 \mu \mathrm{M}$ E4031 exposure in the absence of isoproterenol (ISO; Fig. 4A, B, Movie S3). The presence of EADs contributed to a dramatic APD increase ( $275.2 \pm 36 \mathrm{~ms}$ vs. $653.3 \pm 167.3 \mathrm{~ms}$; Fig. 4C). E4031 prolonged APD in a dose-dependent manner, and in some batches, additional beta-adrenergic stimulation using $100 \mathrm{nM}$ ISO was needed to trigger EADs (Supplemental Fig. 3). Importantly, ISO alone shortened APDs and did not evoke EADs without E4031 (Supplemental Fig. 4A). We further tested blockade of the outward potassium current $I_{\text {to }}$ (responsible for phase 1 repolarization) with 4-Aminopyridine (4-AP) and stimulation of L-type calcium channels (responsible for maintaining phase 2 plateau) with the agonist BayK8644, and both prolonged APDs (Supplemental Fig. 4B, C). These results support that cardiac microtissues using hiPSC-CMs and hCFs are an effective platform for screening pro-arrhythmic toxicants. 
We further investigated how well this human cardiac microtissue model identifies compounds used medically and already determined to be low risk for arrhythmia in clinically-validated doses in patients even when there is a known capacity to block the hERG channel. One such compound is ranolazine, which is a known hERG channel blocker that has therapeutic value as an anti-arrhythmic drug at mean steady-state blood serum concentrations $\left(C_{\max }\right)$ of $6 \mu \mathrm{M}(2600 \mathrm{ng} / \mathrm{mL})$ with $95 \%$ confidence limits of 1 and $14 \mu \mathrm{M}$ (400 and $6100 \mathrm{ng} / \mathrm{mL}$, respectively). ${ }^{62}$ In this concentration range, ranolazine had a minimal effect on APD (Fig. 4D-F), which is in stark contrast to the specific hERG $\left(I_{\mathrm{Kr}}\right)$ blocker E4031 (Fig. 4A-C).

Figure 5 shows a more complete assessment of the dose-dependent effects of ranolazine at $0,2,10$, and $100 \mu \mathrm{M}$ concentrations on eight AP metrics, and traditional dose response curves are shown in Supplemental Fig. 5A. Figure 5A shows representative examples of AP traces. Figure 5B shows a color map presentation of seven of the AP metrics from 35 microtissues, and group data are shown for each of them plus excitability in Fig. 5C. Ranolazine at therapeutic concentrations $(2-10 \mu \mathrm{M})$ had statistically significant effects but very small on all AP metrics (Fig. 5C; see Supplemental Table 1 (top) for complete statistical analysis). For example, average $A \mathrm{AP}_{\mathrm{MxR}}$ changes were negligible (- $9 \mathrm{~ms}$ and $+15 \mathrm{~ms}$ at 2 and $10 \mu \mathrm{M}$, respectively) versus control $0 \mu \mathrm{M}$ (Fig. 5C, F). However, at high concentration (100 $\mu \mathrm{M}$ ranolazine), $A P D_{M X R}$ more than doubled $(\triangle A P D=357 \mathrm{~ms}$, Fig. $5 C, E)$. Despite prolongation, no EADs were observed with ranolazine even in the presence of ISO (see Fig. 5A bottom trace; 1-2 molds per batch, 2 batches) in contrast to the highly selective hERG channel blocker, E4031 (Fig. 4A, B and Supplemental Fig. 3). $100 \mu \mathrm{M}$ ranolazine reduced excitability from $97.1 \%$ at $0-10 \mu \mathrm{M}$ to $85.1 \%$ at $100 \mu \mathrm{M}$, as some of the pacing beats were missed (Fig. 5A-C) and was associated with increased stimulation delay and AP upstroke rise time (Fig. 5B-D) consistent with its therapeutic effects on $I_{\mathrm{Na}}$. Statistically significant changes are detected across all metrics and often at all concentrations of ranolazine (Supplemental Table 1), suggesting that this platform is highly sensitive.

\section{Screening pro-arrhythmic toxicity of environmental toxicant exposure}

We investigated whether acute exposure to the environmental pollutant bisphenol A (BPA) alters AP parameters using our human cardiac microtissue platform. BPA has previously been shown to affect the cardiovascular system, ${ }^{63}$ but its specific effects on arrhythmia are not well characterized. The response of cardiac microtissues to BPA was complex, altering multiple AP parameters including excitability, stimulation time delay, APDs at different levels of repolarization, $A P D_{M x R}$, and $A P D_{\text {tri }}$ in a dose-dependent manner (Fig. 6). A small but significant increase in APD metrics appeared at the lowest concentration of BPA (1 nM), but BPA gradually shortened APD metrics at higher concentrations (Fig. 6B, C). This biphasic response was most pronounced for $A P D_{M \times R}$, which increased by 10.5 ms with $1 \mathrm{nM} B P A$, was similar to no BPA with $10 \mathrm{nM} \mathrm{BPA}$, and decreased by $14.2 \mathrm{~ms}$ and $57.5 \mathrm{~ms}$ with 100 and $1000 \mathrm{nM}$ BPA (Fig. 6C, F). Similar to APD, a biphasic effect of BPA was observed in a fraction of the microtissues ( 6 of 35) on stimulation time delay (Fig. 6B, red/orange boxes at $1 \mathrm{nM}$ in Stim $\Delta$ column), suggesting that complex interactions of BPA on multiple cardiac ion channels involved in both excitation and repolarization was 
revealed during acute exposure across biological replicates in our model. Interestingly, a divergence between $A P D_{80}$ and $A P D_{M \times R}$ was observed at the highest concentration $(1000 \mathrm{nM}$; Fig. 6E, F) due to the appearance of slowed repolarization near the end of AP repolarization (see $1000 \mathrm{nM}$ trace in Fig. 6A), which indicates that very high BPA exposure may also alter inward rectifier current.

The changes in AP metrics were markedly different between ranolazine and BPA, as reflected in the color maps of AP metric changes in response to each compound (Figs. 5B and 6B, respectively). These drug response signatures are visualized in Fig. 7, where significant changes (red, increased; blue, decreased) and their magnitude (color intensity) of the average AP metrics are shown for each compound at indicated concentrations (see also Supplemental Table 2). Ranolazine has a monophasic increase of rise time and $A P D_{30 / 50 / 80}$, whereas $A P D_{M \times R}$ and $A P D_{\text {tri }}$ are biphasic, suggesting nuanced dose-dependent effects (Fig. 7A,C). These results align well with the known clinical effects at low doses and the drug label warnings of fatal arrhythmias (which appear at high doses). In contrast, BPA shows a biphasic response in $\mathrm{APD}_{30 / 50 / 80}$ over the concentration range tested (Fig. 7B). Toward understanding the role of hERG channel blockade in these contrasting results, we applied computer simulations to our system. HERG channel blockade has been shown to increase triangulation of AP (APD $\left.D_{t r i}\right)$ since $I_{\mathrm{Kr}}$ peaks during phase 3 repolarization. Our experimental data and computer simulations in Supplemental Fig. 6 show that E4031 (or $\mathrm{I}_{\mathrm{Kr}}$ blockade in computer modeling) increases the slope of $A P D_{\text {tri }}$ vs. $A P D_{M \times R}$ while $I_{\text {to }}$ block by 4-AP or augmentation of $I_{C a}$ by BayK8644 does not alter the slope of $A P D_{\text {tri }}$ vs. $A P D_{M x R}$, supporting that $A P D_{\text {tri }}$ is an important indicator of hERG channel blockade. Ranolazine increases the slope of $A P D_{\text {tri }}$ Vs. APD ${ }_{M \times R}$ associated with significant APD prolongation at $100 \mu \mathrm{M}$ (Fig. 7C), indicating that hERG blockade becomes significant at this concentration. However, BPA shows biphasic effects on $A P D_{t r i} V_{s}$. APD ${ }_{M \times R}$ increasing the slope in the range of $1-100 \mathrm{nM}$ but not at $1000 \mathrm{nM}$. This suggests that BPA potentially blocks hERG starting at very low concentration (1-10 nM) but later alters other ion channels to mask hERG channel blockade and overall these integrated ion channel changes shorten APD. The results presented here support the notion a contention that our 3D human cardiac microtissue model and comprehensive data analysis provide important information regarding cardiotoxicity of unknown compounds.

\section{Discussion}

In this study, we address a critical need in risk assessment of compounds for pro-arrhythmic cardiotoxicity with a 3D human cardiac microtissue model containing purified hiPSC-derived cardiomyocytes and human cardiac fibroblasts. We show robust quantification of eight distinct parameters derived from the action potential waveform and qualify our NAM by confirming physiological responses and differentiation between the high-risk compound E4031 and low-risk drug ranolazine. Importantly, this differentiation enables reduction of false-positive results, as this 3D microtissue model integrates all ion channels and signals in the human cardiomyocytes. Cardiotoxicity can arise from a variety of aberrant signaling so predictive platforms must be suited for situations in which the mechanism of action is not well defined. ${ }^{53}$ Testing of a compound with poorly described or unknown 
cardiac effects such as BPA reveals nuanced responses (Fig. 6), creating a drug response signature, which enables arrhythmic risk and safety evaluation that yields insights into mechanisms of action and is relevant to physiological metrics of health.

Successful pro-arrhythmic drug screening should be able to detect changes in action potential shapes associated with increased triggered activity (EADs and DADs). A major challenge in developing a predictive risk assessment platform is that multiple factors can contribute to initiation of cardiac arrhythmias, ${ }^{64-68}$ necessitating that the platform be able to capture multiple pro-arrhythmic changes in AP shape. Here, we report the ability to detect altered excitability (\% of captured beats), AP initiation and upstroke, beat-to-beat APD stability, and triangulation of AP shape as well as more standard metrics of prolongation of APD and EAD activity that indicate very high risk for arrhythmia. Our platform goes beyond what is reported by other platforms (such as spontaneous beat rate) that focus on cellular toxicity using live/dead, mitochondrial, and ER imaging, ${ }^{38}$ RNAseq for gene expression, ${ }^{69}$ or contractile amplitude and kinetics from microtissue edge detection ${ }^{70}$ rather than arrhythmia. Due to our high temporal resolution and signal-to-noise ratio, just a single voltage recording without signal averaging can be used to analyze APD metrics and even detect beat-to-beat instability in APD. These parameters can be compared on the same microtissue before and after compound exposure (20 min) using a paired t-test, which increases statistical power and reduces the number of experiments. Other platforms report the calcium transient, using it as a surrogate metric for the voltage signal, but unlike our approach, this surrogate does not provide mechanistic clues underlying arrhythmia induction. The AP metrics defined in this study are more numerous and less variable than those reported from 2D monolayer assays (using voltage/calcium dyes or microelectrode arrays), ${ }^{47,49,50} 3 \mathrm{D}$ spheroid assays, ${ }^{45}$ or engineered heart tissues (EHTs). ${ }^{43,44}$

The 3D microenvironment of our microtissues has several advantages to provide quantitative data for predicting pro-arrhythmic toxicity of chemical compounds. The AP recordings here are the averaged behavior from 12,000-24,000 cells that can mask individual cell-to-cell variation to increase robustness of the response. Triggered activity is thought to be difficult to initiate in the 3D environment due to electrotonic dissipation of depolarizing currents during EADs, known as 'source-sink mismatch.' ${ }^{71}$ Therefore, in order to initiate triggered activity, a large number of cells should independently elicit EADs/DADs simultaneously. Our 3D microtissues have a sufficient number of CMs and do generate EADs (Fig. 4 and Supplemental Fig. 3), suggesting that this model replicates the true risks of EAD formation with its multi-cellular 3D environment as is present at the organ level in the human heart. In a direct comparison of the impact of microenvironment on the predictive capacity of an in vitro model using hESC-CMs, Archer et al. showed with a ROC analysis of drug responses to structural cardiotoxins that a $3 \mathrm{D}$ microtissue platform has increased specificity versus the same measurements made in 2D monolayers. ${ }^{38}$

Our 3D human cardiotoxicity model has several advantages that enable customization and robust proarrhythmic risk evaluation. These advantages are: 1) experimentally determined cell ratios and cell 
phenotypes (Fig. 1B); 2) self-assembly with organotypic heterocellular interspersion (Fig. 1D) while eliminating unnatural substrate or matrix stiffness; 3) robust 3D electrical coupling; 4) rapid optical measurements to track AP shape for highly accurate quantitative metric extraction (Fig. 1E-H); and 5) generation and functional analysis of a large number of individual but consistent microtissues to provide greater throughput and high statistical power in analyses (Figs. 2C, 3D). We have carefully considered the biology of the cardiac cells and employed culturing techniques like moderately longer culture periods and the broadly adopted methods for metabolic selection to purify and mature the hiPSC-CMs, ${ }^{72}$ which demonstrate a more mature ventricular physiology in their electrophysiology compared to unpurified populations. Further, there is evidence that the presence of non-myocytes from hiPSCs or other sources promote more mature electromechanical function. ${ }^{73}$ Incorporation of human cardiac fibroblasts (hCFs) enables heterotypic cell-cell interactions characteristic of the intact myocardium. ${ }^{36,74,75}$ While $5 \%$ primary adult normal hCF content is used for stable, healthy cardiac electromechanical function based on our previous $^{76}$ and current work, the platform allows for alterations in cell composition (such as other cell types, including endothelial cells) and ratios to mimic physiological and pathophysiological conditions that may be important for toxicity evaluation. While our platform can produce hundreds of microtissues in standard cell culture plates, the low variability beat-to-beat, microtissue-to-microtissue, mold-to-mold, and notably hiPSC-CM differentiation batch-to-batch (Fig. 2C and Fig. 3D) reduces the required sample size to tens of microtissues per condition, effectively increasing experimental throughput.

Screening of compounds with diverse and known mechanisms of action is widely accepted as critical practice for assessing the validity of in vitro models, but developing metrics to understand biological mechanisms of action is necessary to move towards screening compounds with unknown mechanisms of action. ${ }^{53}$ Using ranolazine, a well-known effective anti-arrhythmic drug that blocks hERG channel $\left(\mathrm{I}_{\mathrm{Kr}}\right)$ at high concentrations, we show nuanced dose-dependent effects on the AP quantified by our eight metrics (Fig. 5). This altered excitation reflects ranolazine's blockage of multiple ion currents including late $I_{\mathrm{Na}}, \mathrm{I}_{\mathrm{Ca}}, \mathrm{I}_{\mathrm{Na}-\mathrm{Ca}}, \mathrm{I}_{\mathrm{Ks}}$, and $\mathrm{I}_{\mathrm{Kr}} .{ }^{77}$ However, in the clinic, ranolazine is a well-known drug that rarely causes $\mathrm{TdP}$ and is frequently used as anti-arrhythmic treatment despite label warnings of QT prolongation, ${ }^{62}$ suggesting that cardiotoxicity screening must differentiate between high risk compounds and low risk compounds (that could pass safety standards and provide clinical benefit) as we show for E4031 and ranolazine, respectively (Fig. 4). To clearly and quantitatively evaluate arrhythmic risk, we further analyzed dose-dependent changes in triangulation of the AP, APD tri (defined by $A P D_{M x R}-A P D_{50}$ ), which has been implicated as a pro-arrhythmic predictor. ${ }^{78-80}$ Previous studies indicate that an increase in $A P D_{\text {tri }}$ may originate from blockade of either $\mathrm{I}_{\mathrm{Kr}}$ or $\mathrm{I}_{\mathrm{Ks}}$, resulting in delayed phase 3 repolarization. ${ }^{78,81}$ Our data also showed that specific $I_{\mathrm{Kr}}$ blockade by E4031 increases $\mathrm{APD}_{\text {tri }}$ but that $\mathrm{I}_{\text {to }}$ blockade by 4-AP or $\mathrm{I}_{\mathrm{Ca}}$ activation by BayK8644 had no effect on $\mathrm{APD}_{\text {tri, }}$ which we confirm with computer modeling (Supplemental Fig. 6). The trend of increasing APD tri with a similarly increasing APD would suggest risky $\mathrm{I}_{\mathrm{Kr}}$ block and can be visualized by plotting $A P D_{\text {tri }}$ versus $A P D_{M \times R}$. The slope of the regression line thus increases when $\mathrm{I}_{\mathrm{Kr}}$ is blocked and is a clear indicator for dose-dependent arrhythmic toxicity. At lower concentration, ranolazine did not affect slope but at $100 \mu \mathrm{M}$ it significantly increased (Fig. 7C, bar plot), 
suggesting that ranolazine blocks $\mathrm{I}_{\mathrm{K} r}$, which is in agreement with previous voltage clamp studies that found $\mathrm{IC}_{50}$ is $11.5 \mu \mathrm{M}$ for $\mathrm{I}_{\mathrm{Kr}}{ }^{77}$ However, the less well-known compound BPA showed a biphasic effect on slope of the $A P D_{\text {tri }}$ Vs. $A P D_{M \times R}$ relationship, peaking at $10 \mathrm{nM}$ and decreasing at higher concentrations, while $A P D_{M x R}$ decreases (Fig. 7D). These data suggest that $I_{K r}$ may be affected at BPA concentrations starting at very low concentration $(10 \mathrm{nM})$ and yet other ion channels and currents may compensate to counteract this effect at higher concentrations. Ultimately, this integrated AP response in human 3D cardiac microtissues with hiPSC-CMs is a defining feature of our model and a significant advantage to be able to predict pro-arrhythmic cardiotoxicity with high sensitivity that will be useful for targeted pharmaceutical development earlier in the drug development pipeline or predictive IVIVE to define safe chemical exposure levels.

It was our aim with this study and in our human cardiotoxicity tissue-engineered model to provide a fitfor-purpose NAM for advancing toxicity risk assessment for pharmaceutical development and safety evaluation of chemicals and environmental toxicants while reducing use of animals, improving mechanistic understanding of cardiotoxicity, and facilitating broad adoption of this simple human in vitro platform. Our platform showed expected changes in arrhythmia metrics to known toxicants and revealed alterations in response to the endocrine disrupting chemical BPA with high sensitivity that suggest cardiac risk and point toward disrupted ion channels as mechanisms of action that may not have been detected in other platforms with more surrogate metrics. Cardiotoxicity risk likely depends on multiple factors including sex, race, and disease status, ${ }^{82}$ and toxic effects may be observed to a lesser degree in healthy human subjects compared to patients with comorbidities, defined as 'hidden toxicity'. ${ }^{18}$ These diverse risk factors can be incorporated into our 3D microtissue risk assessment platform through the use of different hiPSC lines; thus exciting opportunities exist to better understand many fundamental mechanisms of toxicity. The predictive value of this platform has the potential to accelerate broad adoption and advances in the regulatory landscape for cardiotoxicity evaluation.

\section{Methods}

\section{Cardiomyocyte Differentiation.}

Cardiomyocytes (CMs) were differentiated from human induced pluripotent stem cells (hiPSCs; Gibco human female episomal iPSCs) in high-density monolayer cultures using CDM3 medium ${ }^{83}$ and Wnt signal activation and inhibition ${ }^{84}$ (Fig. 1A). Briefly, hiPSCs were treated with $6 \mu$ M Chiron 99021 (Tocris), a glycogen synthase kinase 3 (GSK3) inhibitor at day 1, followed by $5 \mu$ M IWP2 (Tocris), a chemical Wnt inhibitor at day 3. Cardiac phenotype, expressed by beating cells, was visible between days 8 and 12 and upon beating, cardiomyocytes were cultured in RPMI 1640 medium with B27 supplement (RPMI + B27; Gibco). Cardiomyocytes differentiated from hiPSCs were used for the production of microtissues between days 14 and 18 of differentiation or were further purified with a lactate protocol. Cardiomyocytes designated for lactate purification were harvested and replated to new culture vessels coated with Matrigel ${ }^{\circledR}$ (Corning) in RPMI + B27. These cells were deprived of media change for 4 days and were fed 
with lactate media (DMEM without glucose, L-glutamine, phenol red, sodium pyruvate and sodium bicarbonate (D-5030, Sigma) + 4 mM L-glutamine, 1X Non-Essential Amino Acids, 1x Glutamax and $4 \mathrm{mM}$ lactate, $\mathrm{pH}$ 7.4). Lactate purified cells were fed with RPMI + B27 + 1\% penicillin/streptomycin (P/S). Cardiac purity was measured by flow cytometry analysis as previously described. ${ }^{85}$

\section{Human Cardiac Fibroblast Culture.}

Commercially available human cardiac fibroblasts (hCFs, Sigma) were maintained and passaged in DMEM/F12 supplemented with $10 \%$ fetal bovine serum (FBS), $1 \%$ penicillin/streptomycin (P/S), and $4 \mathrm{ng} / \mathrm{ml}$ basic fibroblast growth factor (Reprocell). hCFs were incorporated into cardiac microtissues at cell passages $\mathrm{P} 2-\mathrm{P} 4$.

\section{Fabrication of Hydrogels and 3D Culture.}

Scaffold-free 3D spherical microtissues were generated using non-adhesive agarose gels with cylindrical microwells with hemispherical bottoms to guide self-assembly (Fig. 1B). Sterilized $2 \%$ (wt/vol) agarose was pipetted into molds designed for 24-well plates with 800- $\mu \mathrm{m}$-diameter rounded pegs (3D Petri Dish ${ }^{\circledR}$, MicroTissues). After being cooled to room temperature ( $5 \mathrm{~min})$, the agarose gels were separated from the molds and transferred to single wells of 24 -well plates. For equilibration, $1 \mathrm{~mL} \mathrm{RPMI}+\mathrm{B} 27+1 \% \mathrm{P} / \mathrm{S}$ medium was added to each well. Hydrogels were equilibrated for at least $1 \mathrm{hr}$ at $37^{\circ} \mathrm{C}$ in a humidified incubator with $5 \% \mathrm{CO}_{2}$. Molds were transferred to 6-well plates for electrical stimulation, and hiPSC-CMs with or without additional $5 \% \mathrm{hCF}$ in suspension were added to the center of the hydrogel seeding chamber (420-840K cells/mold in 35 recesses) and allowed to settle into the recesses for $30 \mathrm{~min}$. Medium (RPMI + B27 with 1\% P/S and 10\% FBS with $5 \mu \mathrm{M}$ rock inhibitor (Y27632)) was then added to each well $(5 \mathrm{~mL})$. Medium was changed to RPMI + B27 with $1 \% \mathrm{P} / \mathrm{S}$ and $10 \% \mathrm{FBS}$ at $24-48 \mathrm{hr}$, and cells were cultured for 6-8 days with media changes every other day. During the 3D culture period, the selfassembled microtissues were field stimulated with a $1 \mathrm{~Hz}, 10.0 \mathrm{~V}, 4.0 \mathrm{~ms}$ duration bipolar pulse train.

\section{Microtissue Size Analysis.}

Stitched $4 \mathrm{X}$ phase-contrast images of whole 24-well microtissue hydrogels were captured with a Nikon TE2000-U and a black and white/color digital camera (MicroVideo Instruments, Avon, MA). NIS Elements software was used for image acquisition and analysis. Image thresholding and particle size analysis was performed to determine the top view cross-sectional area of individual microtissues across each mold.

\section{D Tissue Sections and Immunohistochemistry.}

Microtissues in 24-well hydrogels were fixed with 4\% (vol/vol) paraformaldehyde (Electron Microscopy Sciences, Hatfield, PA) and $8 \%(\mathrm{wt} / \mathrm{vol})$ sucrose in PBS overnight at room temperature. Molds were then rinsed twice with PBS and fully equilibrated (as indicated by their sinking, usually over $12 \mathrm{hrs}$ ) with $15 \%$ and then $30 \%(\mathrm{wt} / \mathrm{vol})$ sucrose in PBS. Whole agarose gels containing microtissues were removed from sucrose solution, blotted dry, and embedded in Tissue-Tek CRYO-OCT Compound (Sakura). Blocks were 
stored at $-80^{\circ} \mathrm{C}$, sectioned on a Leica CM3050 cryostat microtome (Leica Biosystems, Buffalo Grove, IL) into 10- $\mu \mathrm{m}$-thick sections, and placed on Superfrost Plus slides. After being air dried for $15 \mathrm{~min}$, sections were post-fixed in $4 \%$ paraformaldehyde in PBS. For immunofluorescent staining at room temperature, frozen sections were rinsed 3 times for 5 min with 1X PBS wash buffer. Non-specific binding was blocked with $1.5 \%$ goat serum for $1 \mathrm{hr}$, followed by $24 \mathrm{hr}$ incubation in primary and followed by a $1 \mathrm{hr}$ incubation in secondary antibodies diluted in $1.5 \%$ goat serum. Primary antibodies were directed against cardiac troponin I (cTnl, 1:100, Abcam ab47003) and vimentin (1:100, Sigma V6630), and secondary antibodies were conjugated to Alexa Fluor 488 or Alexa Fluor 594 (1:200, Invitrogen). Coverslips were mounted with Vectashield mounting medium with DAPI. Images were taken with an Olympus FV3000 Confocal Microscope and processed using ImageJ.

\section{Optical Mapping and Automated Action Potential Analysis.}

Microtissues were loaded with voltage-sensitive di-4-ANEPPS $\left(5 \mu \mathrm{M}\right.$ for $10 \mathrm{~min}$ at $\left.35^{\circ} \mathrm{C}\right)$ for measurements of membrane potential $\left(V_{\mathrm{m}}\right)$. Fluorescence images were acquired at $979 \mathrm{frames} / \mathrm{s}$ using a Photometrics Evolve +128 EMCCD camera $\left(2 \times 2\right.$ binning to $64 \times 64$ pixels, $18.7 \times 18.7-\mu \mathrm{m}^{2}$ resolution, $1.2 \times 1.2-\mathrm{mm}^{2}$ field of view) and an Olympus MXV10 macroview optical system. ${ }^{86}$ Typically, four microtissues were recorded simultaneously per scan at this magnification. A step-by-step illustration of automated data analysis is available in Supplemental Fig. 1. Briefly, the pixels with APs were identified from Fast Fourier transformation (FFT) of fluorescence signals. After appropriate thresholding and image segmentation, the region of each microtissue was grouped and the fluorescence signals from the pixels in the same microtissue were average and used for AP analysis (Supplemental Fig. 1).

\section{Validation and Screening of Toxicants for Arrhythmogenic Risk.}

A single mold of microtissues was mounted on a temperature-controlled chamber (Dual Automatic Temperature Controller TC-344B, Warner Instrument) to maintain $35 \pm 1{ }^{\circ} \mathrm{C}$ and bathed with Tyrode's solution containing (in mM) $140 \mathrm{NaCl}, 5.1 \mathrm{KCl}, 1 \mathrm{MgCl}_{2}, 1 \mathrm{CaCl}_{2}, 0.33 \mathrm{NaH}_{2} \mathrm{PO}_{4}, 5 \mathrm{HEPES}$, and 7.5 glucose. Microtissues were stimulated with a platinum field stimulation electrode (Myopacer EP field stimulator, lonOptix, Milton, MA). The test compounds including E4031, 4-AP, BayK8644, ISO were purchased from Sigma Aldrich and dissolved in 100\% DMSO to prepare 0.01-0.5 mM stock solutions. BPA was dissolved in $10 \%$ ethanol stock solution and diluted in Tyrode solution to the final concentration. Microtissues were exposed to vehicle (DMSO or ethanol) and the indicated concentrations of test compounds for $20 \mathrm{~min}$, and action potentials were measured as described above.

\section{Statistical Analyses.}

The data from optical mapping are expressed as mean \pm SD for $n$ microtissues unless otherwise indicated. Statistical analyses were performed using Student's two tailed paired and unpaired t-test. $P$ values of 0.05 were considered statistically significant. Normality test was done using Kolmogorov- 
Smirnov test. The test for the equality of regression coefficients was done using Z statistics of two slopes and SE as described. ${ }^{87,88}$

\section{Declarations}

\section{Acknowledgements:}

Funding is gratefully acknowledged from NIH U01 ES028184.

\section{Author Contributions:}

CMK, TYK, UM, BRC, and KLKC conceived and designed research; CMK, TYK, FM, AHS, RJK, and BRC performed experiments; CMK, TYK, UM, BRC, and KLKC analyzed data and interpreted results of experiments; CMK, UM, BRC, and KLKC drafted and edited manuscript; CMK, TYK, FM, AHS, RJK, UM, BRC, and KLKC approved final version of manuscript.

\section{References}

1. Abbott, G. W. \& Roepke, T. K. Pharmacogenetics of drug-induced arrhythmias. Expert Rev Clin Pharmacol. 1, 93-104 https://doi.org/10.1586/17512433.1.1.93 (2008).

2. Barnes, B. J. \& Hollands, J. M. Drug-induced arrhythmias. Crit Care Med. 38, S188-197 https://doi.org/10.1097/CCM.0b013e3181de112a (2010).

3. Bossu, A., van der Heyden, M. A., de Boer, T. P. \& Vos, M. A. A 2015 focus on preventing drug-induced arrhythmias. Expert Rev Cardiovasc Ther. 14, 245-253 https://doi.org/10.1586/14779072.2016.1116940 (2016).

4. Fenichel, R. R. et al. Drug-induced torsades de pointes and implications for drug development. $J$ Cardiovasc Electrophysiol. 15, 475-495 https://doi.org/10.1046/j.1540-8167.2004.03534.x (2004).

5. Lee, A. \& Pickham, D. Basic Cardiac Electrophysiology and Common Drug-induced Arrhythmias. Crit Care Nurs Clin North Am. 28, 357-371 https://doi.org/10.1016/j.cnc.2016.04.007 (2016).

6. Recanatini, M., Poluzzi, E., Masetti, M., Cavalli, A. \& De Ponti, F. QT prolongation through hERG K(+) channel blockade: current knowledge and strategies for the early prediction during drug development. Med Res Rev. 25, 133-166 https://doi.org/10.1002/med.20019 (2005).

7. Haverkamp, W. et al. The potential for QT prolongation and pro-arrhythmia by non-anti-arrhythmic drugs: clinical and regulatory implications. Report on a Policy Conference of the European Society of Cardiology. Cardiovasc Res 47, 219-233, doi:10.1016/s0008-6363(00)00119-x (2000).

8. Prüss-Üstün, A. \& Corvalán, C. Preventing disease through healthy environments: towards an estimate of the environmental burden of disease. Vol. 12(SciELO Brasil, 2006).

9. Virani, S. S. et al. Heart Disease and Stroke Statistics-2020 Update: A Report From the American Heart Association. Circulation. 141, e139-e596 https://doi.org/10.1161/CIR.0000000000000757 (2020). 
10. Tanner, M. R. \& Beeton, C. Differences in ion channel phenotype and function between humans and animal models. Front Biosci (Landmark Ed). 23, 43-64 https://doi.org/10.2741/4581 (2018).

11. Bracken, M. B. Why animal studies are often poor predictors of human reactions to exposure. $J R$ Soc Med. 102, 120-122 https://doi.org/10.1258/jrsm.2008.08k033 (2009).

12. Haraguchi, Y., Ohtsuki, A., Oka, T. \& Shimizu, T. Electrophysiological analysis of mammalian cells expressing hERG using automated 384-well-patch-clamp. BMC Pharmacol Toxicol. 16, 39 https://doi.org/10.1186/s40360-015-0042-9 (2015).

13. Wacker, S. \& Noskov, S. Y. Performance of Machine Learning Algorithms for Qualitative and Quantitative Prediction Drug Blockade of hERG1 channel. Comput Toxicol. 6, 55-63 https://doi.org/10.1016/j.comtox.2017.05.001 (2018).

14. Alinejad, S., Kazemi, T., Zamani, N., Hoffman, R. S. \& Mehrpour, O. A systematic review of the cardiotoxicity of methadone. EXCLI J. 14, 577-600 https://doi.org/10.17179/excli2015-553 (2015).

15. Heranval, A. et al. Drugs with potential cardiac adverse effects: Retrospective study in a large cohort of parkinsonian patients. Rev Neurol (Paris). 172, 318-323 https://doi.org/10.1016/j.neurol.2015.11.007 (2016).

16. Sun, C., Brice, J. A. \& Clark, R. F. Brugada-Type Pattern on Electrocardiogram Associated with HighDose Loperamide Abuse. J Emerg Med. 54, 484-486 https://doi.org/10.1016/j.jemermed.2017.12.040 (2018).

17. Ramalho, D. \& Freitas, J. Drug-induced life-threatening arrhythmias and sudden cardiac death: A clinical perspective of long QT, short QT and Brugada syndromes. Rev Port Cardiol. 37, 435-446 https://doi.org/10.1016/j.repc.2017.07.010 (2018).

18. Ferdinandy, P. et al. Definition of hidden drug cardiotoxicity: paradigm change in cardiac safety testing and its clinical implications. Eur Heart J. 40, 1771-1777 https://doi.org/10.1093/eurheartj/ehy365 (2019).

19. Singh, B. N. Amiodarone: a multifaceted antiarrhythmic drug. Curr Cardiol Rep. 8, 349-355 https://doi.org/10.1007/s11886-006-0074-2 (2006).

20. Singh, B. N. \& Wadhani, N. Antiarrhythmic and proarrhythmic properties of QT-prolonging antianginal drugs. J Cardiovasc Pharmacol Ther. 9 Suppl (1), S85-97 https://doi.org/10.1177/107424840400900107 (2004).

21. Wu, L. et al. Augmentation of late sodium current unmasks the proarrhythmic effects of amiodarone. Cardiovasc Res. 77, 481-488 https://doi.org/10.1093/cvr/cvm069 (2008).

22. Kurokawa, Y. K. \& George, S. C. Tissue engineering the cardiac microenvironment: Multicellular microphysiological systems for drug screening. Adv Drug Deliv Rev. 96, 225-233 https://doi.org/10.1016/j.addr.2015.07.004 (2016).

23. Strickland, J. et al. Status of acute systemic toxicity testing requirements and data uses by U.S. regulatory agencies. Regul Toxicol Pharmacol. 94, 183-196 https://doi.org/10.1016/j.yrtph.2018.01.022 (2018). 
24. Methods, I. C. C. o. t. V. o. A. A Strategic Roadmap for Establishing New Approaches to Evaluate the Safety of Chemicals and Medical Products in the United States. doi:10.22427/NTP-ICCVAMROADMAP2018 (2018).

25. Parish, S. T. et al. An evaluation framework for new approach methodologies (NAMs) for human health safety assessment. Regul Toxicol Pharmacol. 112, 104592 https://doi.org/10.1016/j.yrtph.2020.104592 (2020).

26. Sager, P. T., Gintant, G., Turner, J. R., Pettit, S. \& Stockbridge, N. Rechanneling the cardiac proarrhythmia safety paradigm: a meeting report from the Cardiac Safety Research Consortium. Am Heart J. 167, 292-300 (2014).

27. Strauss, D. G. et al. Comprehensive In Vitro Proarrhythmia Assay (CiPA) Update from a Cardiac Safety Research Consortium / Health and Environmental Sciences Institute / FDA Meeting. Ther Innov Regul Sci. 53, 519-525 https://doi.org/10.1177/2168479018795117 (2019).

28. Gintant, G., Sager, P. T. \& Stockbridge, N. Evolution of strategies to improve preclinical cardiac safety testing. Nat Rev Drug Discov. 15, 457-471 https://doi.org/10.1038/nrd.2015.34 (2016).

29. Chen, I. Y., Matsa, E. \& Wu, J. C. Induced pluripotent stem cells: at the heart of cardiovascular precision medicine. Nat Rev Cardiol. 13, 333-349 https://doi.org/10.1038/nrcardio.2016.36 (2016).

30. Gintant, G. et al. Use of Human Induced Pluripotent Stem Cell-Derived Cardiomyocytes in Preclinical Cancer Drug Cardiotoxicity Testing: A Scientific Statement From the American Heart Association. Circ Res. 125, e75-e92 https://doi.org/10.1161/RES.0000000000000291 (2019).

31. Sinnecker, D., Laugwitz, K. L. \& Moretti, A. Induced pluripotent stem cell-derived cardiomyocytes for drug development and toxicity testing. Pharmacol Ther. 143, 246-252 https://doi.org/10.1016/j.pharmthera.2014.03.004 (2014).

32. Blinova, K. et al. International Multisite Study of Human-Induced Pluripotent Stem Cell-Derived Cardiomyocytes for Drug Proarrhythmic Potential Assessment. Cell Rep. 24, 3582-3592 https://doi.org/10.1016/j.celrep.2018.08.079 (2018).

33. Nguyen, D. C. et al. Microscale generation of cardiospheres promotes robust enrichment of cardiomyocytes derived from human pluripotent stem cells. Stem Cell Reports. 3, 260-268 https://doi.org/10.1016/j.stemcr.2014.06.002 (2014).

34. Beauchamp, P. et al. Development and Characterization of a Scaffold-Free 3D Spheroid Model of Induced Pluripotent Stem Cell-Derived Human Cardiomyocytes. Tissue Eng Part C Methods. 21, 852861 https://doi.org/10.1089/ten.TEC.2014.0376 (2015).

35. Desroches, B. R. et al. Functional scaffold-free 3-D cardiac microtissues: a novel model for the investigation of heart cells. Am J Physiol Heart Circ Physiol. 302, H2031-2042 https://doi.org/10.1152/ajpheart.00743.2011 (2012).

36. Kofron, C. M. et al. Gq-activated fibroblasts induce cardiomyocyte action potential prolongation and automaticity in a three-dimensional microtissue environment. Am J Physiol Heart Circ Physiol. 313, H810-H827 https://doi.org/10.1152/ajpheart.00181.2017 (2017). 
37. Polonchuk, L. et al. Cardiac spheroids as promising in vitro models to study the human heart microenvironment. Sci Rep. 7, 7005 https://doi.org/10.1038/s41598-017-06385-8 (2017).

38. Archer, C. R. et al. Characterization and Validation of a Human 3D Cardiac Microtissue for the Assessment of Changes in Cardiac Pathology. Sci Rep. 8, 10160 https://doi.org/10.1038/s41598018-28393-y (2018).

39. Verheijen, M. et al. Bringing in vitro analysis closer to in vivo: Studying doxorubicin toxicity and associated mechanisms in 3D human microtissues with PBPK-based dose modelling. Toxicol Lett. 294, 184-192 https://doi.org/10.1016/j.toxlet.2018.05.029 (2018).

40. Ravenscroft, S. M., Pointon, A., Williams, A. W., Cross, M. J. \& Sidaway, J. E. Cardiac Non-myocyte Cells Show Enhanced Pharmacological Function Suggestive of Contractile Maturity in Stem Cell Derived Cardiomyocyte Microtissues. Toxicol Sci. 152, 99-112 https://doi.org/10.1093/toxsci/kfw069 (2016).

41. Pointon, A. et al. From the Cover: High-Throughput Imaging of Cardiac Microtissues for the Assessment of Cardiac Contraction during Drug Discovery. Toxicol Sci. 155, 444-457 https://doi.org/10.1093/toxsci/kfw227 (2017).

42. Zhao, Y. et al. A Platform for Generation of Chamber-Specific Cardiac Tissues and Disease Modeling. Cell 176, 913-927 e918, doi:10.1016/j.cell.2018.11.042 (2019).

43. Feric, N. T. et al. Engineered Cardiac Tissues Generated in the Biowire II: A Platform for Human-Based Drug Discovery. Toxicol Sci. https://doi.org/10.1093/toxsci/kfz168 (2019).

44. Takeda, M. et al. Development of In Vitro Drug-Induced Cardiotoxicity Assay by Using ThreeDimensional Cardiac Tissues Derived from Human Induced Pluripotent Stem Cells. Tissue Eng Part C Methods. 24, 56-67 https://doi.org/10.1089/ten.TEC.2017.0247 (2018).

45. Bergstrom, G., Christoffersson, J., Schwanke, K., Zweigerdt, R. \& Mandenius, C. F. Stem cell derived in vivo-like human cardiac bodies in a microfluidic device for toxicity testing by beating frequency imaging. Lab Chip. 15, 3242-3249 https://doi.org/10.1039/c5lc00449g (2015).

46. Sirenko, O. et al. In vitro cardiotoxicity assessment of environmental chemicals using an organotypic human induced pluripotent stem cell-derived model. Toxicol Appl Pharmacol. 322, 60-74 https://doi.org/10.1016/j.taap.2017.02.020 (2017).

47. Jahnke, H. G. et al. A novel 3D label-free monitoring system of hES-derived cardiomyocyte clusters: a step forward to in vitro cardiotoxicity testing. PLoS One. 8, e68971 https://doi.org/10.1371/journal.pone.0068971 (2013).

48. Takasuna, K. et al. Comprehensive in vitro cardiac safety assessment using human stem cell technology: Overview of CSAHi HEART initiative. J Pharmacol Toxicol Methods. 83, 42-54 https://doi.org/10.1016/j.vascn.2016.09.004 (2017).

49. Fleischer, S., Jahnke, H. G., Fritsche, E., Girard, M. \& Robitzki, A. A. Comprehensive human stem cell differentiation in a 2D and 3D mode to cardiomyocytes for long-term cultivation and multiparametric monitoring on a multimodal microelectrode array setup. Biosens Bioelectron. 126, 624-631 https://doi.org/10.1016/j.bios.2018.10.061 (2019). 
50. Asahi, Y. et al. Electrophysiological evaluation of pentamidine and 17-AAG in human stem cellderived cardiomyocytes for safety assessment. Eur J Pharmacol. 842, 221-230 https://doi.org/10.1016/j.ejphar.2018.10.046 (2019).

51. Navarrete, E. G. et al. Screening drug-induced arrhythmia [corrected] using human induced pluripotent stem cell-derived cardiomyocytes and low-impedance microelectrode arrays. Circulation. 128, S3-13 https://doi.org/10.1161/CIRCULATIONAHA.112.000570 (2013).

52. Pistollato, F., Bremer-Hoffmann, S., Healy, L., Young, L. \& Stacey, G. Standardization of pluripotent stem cell cultures for toxicity testing. Expert Opin Drug Metab Toxicol. 8, 239-257 https://doi.org/10.1517/17425255.2012.639763 (2012).

53. Magdy, T., Schuldt, A. J. T., Wu, J. C., Bernstein, D. \& Burridge, P. W. Human Induced Pluripotent Stem Cell (hiPSC)-Derived Cells to Assess Drug Cardiotoxicity: Opportunities and Problems. Annu Rev Pharmacol Toxicol. 58, 83-103 https://doi.org/10.1146/annurev-pharmtox-010617-053110 (2018).

54. Berenfeld, O. \& Efimov, I. Optical Mapping. Card Electrophysiol Clin. 11, 495-510 https://doi.org/10.1016/j.ccep.2019.04.004 (2019).

55. Salama, G. \& Choi, B. R. Imaging ventricular fibrillation. J Electrocardiol. 40, S56-61 https://doi.org/10.1016/j.jelectrocard.2007.06.021 (2007).

56. Nachimuthu, S., Assar, M. D. \& Schussler, J. M. Drug-induced QT interval prolongation: mechanisms and clinical management. Ther Adv Drug Saf. 3, 241-253 https://doi.org/10.1177/2042098612454283 (2012).

57. Achilli, T. M., McCalla, S., Meyer, J., Tripathi, A. \& Morgan, J. R. Multilayer spheroids to quantify drug uptake and diffusion in 3D. Mol Pharm. 11, 2071-2081 https://doi.org/10.1021/mp500002y (2014).

58. Clay, J. R., Ogbaghebriel, A., Paquette, T., Sasyniuk, B. I. \& Shrier, A. A quantitative description of the E4031-sensitive repolarization current in rabbit ventricular myocytes. Biophys J. 69, 1830-1837 https://doi.org/10.1016/S0006-3495(95)80053-6 (1995).

59. Ma, J. et al. High purity human-induced pluripotent stem cell-derived cardiomyocytes: electrophysiological properties of action potentials and ionic currents. Am J Physiol Heart Circ Physiol. 301, H2006-2017 https://doi.org/10.1152/ajpheart.00694.2011 (2011).

60. Taggart, P., Sutton, P. M., Boyett, M. R., Lab, M. \& Swanton, H. Human ventricular action potential duration during short and long cycles. Rapid modulation by ischemia. Circulation. 94, 2526-2534 https://doi.org/10.1161/01.cir.94.10.2526 (1996).

61. Franz, M. R., Swerdlow, C. D., Liem, L. B. \& Schaefer, J. Cycle length dependence of human action potential duration in vivo. Effects of single extrastimuli, sudden sustained rate acceleration and deceleration, and different steady-state frequencies. J Clin Invest. 82, 972-979 https://doi.org/10.1172/JCl113706 (1988).

62. Gilead Sciences, F. et al. (eds) (CA, 2015).

63. Gao, X. \& Wang, H. S. Impact of bisphenol a on the cardiovascular system - epidemiological and experimental evidence and molecular mechanisms. Int J Environ Res Public Health. 11, 8399-8413 https://doi.org/10.3390/ijerph110808399 (2014). 
64. Elson, J. \& Mason, J. W. General concepts and mechanisms of ventricular tachycardia. Cardiol Clin. 4, 459-472 (1986).

65. Ebinger, M. W., Krishnan, S. \& Schuger, C. D. Mechanisms of ventricular arrhythmias in heart failure. Curr Heart Fail Rep. 2, 111-117 (2005).

66. Makati, K. J. et al. Advances in mechanisms of atrial fibrillation: structural remodeling, highfrequency fractionated electrograms, and reentrant AF drivers. J Interv Card Electrophysiol. 23, 4549 https://doi.org/10.1007/s10840-008-9256-8 (2008).

67. Sattler, S. M. et al. Ventricular Arrhythmias in First Acute Myocardial Infarction: Epidemiology, Mechanisms, and Interventions in Large Animal Models. Front Cardiovasc Med. 6, 158 https://doi.org/10.3389/fcvm.2019.00158 (2019).

68. Pellman, J., Zhang, J. \& Sheikh, F. Myocyte-fibroblast communication in cardiac fibrosis and arrhythmias: Mechanisms and model systems. J Mol Cell Cardiol. 94, 22-31 https://doi.org/10.1016/j.yjmcc.2016.03.005 (2016).

69. Richards, D. J. et al. Human cardiac organoids for the modelling of myocardial infarction and drug cardiotoxicity. Nat Biomed Eng. 4, 446-462 https://doi.org/10.1038/s41551-020-0539-4 (2020).

70. Beauchamp, P. et al. 3D Co-culture of hiPSC-Derived Cardiomyocytes With Cardiac Fibroblasts Improves Tissue-Like Features of Cardiac Spheroids. Front Mol Biosci. 7, 14 https://doi.org/10.3389/fmolb.2020.00014 (2020).

71. Xie, Y., Sato, D., Garfinkel, A., Qu, Z. \& Weiss, J. N. So little source, so much sink: requirements for afterdepolarizations to propagate in tissue. Biophys J. 99, 1408-1415 https://doi.org/10.1016/j.bpj.2010.06.042 (2010).

72. Lin, B. et al. Culture in Glucose-Depleted Medium Supplemented with Fatty Acid and 3,3',5-Triiodo-IThyronine Facilitates Purification and Maturation of Human Pluripotent Stem Cell-Derived Cardiomyocytes. Front Endocrinol (Lausanne). 8, 253 https://doi.org/10.3389/fendo.2017.00253 (2017).

73. Tiburcy, M. et al. Defined Engineered Human Myocardium With Advanced Maturation for Applications in Heart Failure Modeling and Repair. Circulation. 135, 1832-1847 https://doi.org/10.1161/CIRCULATIONAHA.116.024145 (2017).

74. Kofron, C. M. \& Mende, U. In vitro models of the cardiac microenvironment to study myocyte and nonmyocyte crosstalk: bioinspired approaches beyond the polystyrene dish. J Physiol. 595, 3891-3905 https://doi.org/10.1113/JP273100 (2017).

75. Zuppinger, C. 3D Cardiac Cell Culture: A Critical Review of Current Technologies and Applications. Front Cardiovasc Med. 6, 87 https://doi.org/10.3389/fcvm.2019.00087 (2019).

76. Rupert, C. E., Kim, T. Y., Choi, B. R. \& Coulombe, K. L. K. Human Cardiac Fibroblast Number and Activation State Modulate Electromechanical Function of hiPSC-Cardiomyocytes in Engineered Myocardium. Stem Cells Int 2020, 9363809, doi:10.1155/2020/9363809 (2020).

77. Antzelevitch, C. et al. Electrophysiological effects of ranolazine, a novel antianginal agent with antiarrhythmic properties. Circulation. 110, 904-910 
https://doi.org/10.1161/01.CIR.0000139333.83620.5D (2004).

78. Guerard, N. C., Traebert, M., Suter, W. \& Dumotier, B. M. Selective block of IKs plays a significant role in MAP triangulation induced by IKr block in isolated rabbit heart. J Pharmacol Toxicol Methods. 58, 32-40 https://doi.org/10.1016/j.vascn.2008.05.129 (2008).

79. Grunnet, M. Repolarization of the cardiac action potential. Does an increase in repolarization capacity constitute a new anti-arrhythmic principle?. Acta Physiol (Oxf). 198 Suppl 676, 1-48 https://doi.org/10.1111/j.1748-1716.2009.02072.x (2010).

80. Hondeghem, L. M., Carlsson, L. \& Duker, G. Instability and triangulation of the action potential predict serious proarrhythmia, but action potential duration prolongation is antiarrhythmic. Circulation. 103, 2004-2013 https://doi.org/10.1161/01.cir.103.15.2004 (2001).

81. Trenor, B. et al. In silico assessment of drug safety in human heart applied to late sodium current blockers. Channels. 7, 249-262 https://doi.org/10.4161/chan.24905 (2013).

82. Zeng, H., Wang, J., Clouse, H., Lagrutta, A. \& Sannajust, F. Resolving the Reversed Rate Effect of Calcium Channel Blockers on Human-Induced Pluripotent Stem Cell-Derived Cardiomyocytes and the Impact on In Vitro Cardiac Safety Evaluation. Toxicol Sci. 167, 573-580 https://doi.org/10.1093/toxsci/kfy264 (2019).

83. Burridge, P. W. et al. A universal system for highly efficient cardiac differentiation of human induced pluripotent stem cells that eliminates interline variability. PLoS One. 6, e18293 https://doi.org/10.1371/journal.pone.0018293 (2011).

84. Lian, X. et al. Robust cardiomyocyte differentiation from human pluripotent stem cells via temporal modulation of canonical Wnt signaling. Proc Natl Acad Sci U S A. 109, E1848-1857 https://doi.org/10.1073/pnas.1200250109 (2012).

85. Rupert, C. E., Irofuala, C. \& Coulombe, K. L. K. Practical adoption of state-of-the-art hiPSCcardiomyocyte differentiation techniques. PLoS One. 15, e0230001 https://doi.org/10.1371/journal.pone.0230001 (2020).

86. Kim, T. Y. et al. Directed fusion of cardiac spheroids into larger heterocellular microtissues enables investigation of cardiac action potential propagation via cardiac fibroblasts. PLoS One. 13, e0196714 https://doi.org/10.1371/journal.pone.0196714 (2018).

87. Cohen, J., Cohen, P., West, S. G. \& Aiken, L. S. Applied multiple regression/correlation analysis for the behavioral sciences 3rd edn (Lawrence Erlbaum Associates, Publishers, 2003).

88. Paternoster, R., Brame, R., Mazerolle, P. \& Piquero, A. R. Using the Correct Statistical Test for the Equality of Regression Coefficients. Criminology. 36 (4), 859-866 (1998).

\section{Figures}


Experimental Timeline:
A Day 03

$\sum$ differentiate
self-assemble
Lactate purify + mature
3D culture

\section{D Culture Platform:}

B hiPSC-CMs $+5 \% \mathrm{hCF}$

(12,000-24,000 cells/microtissue)
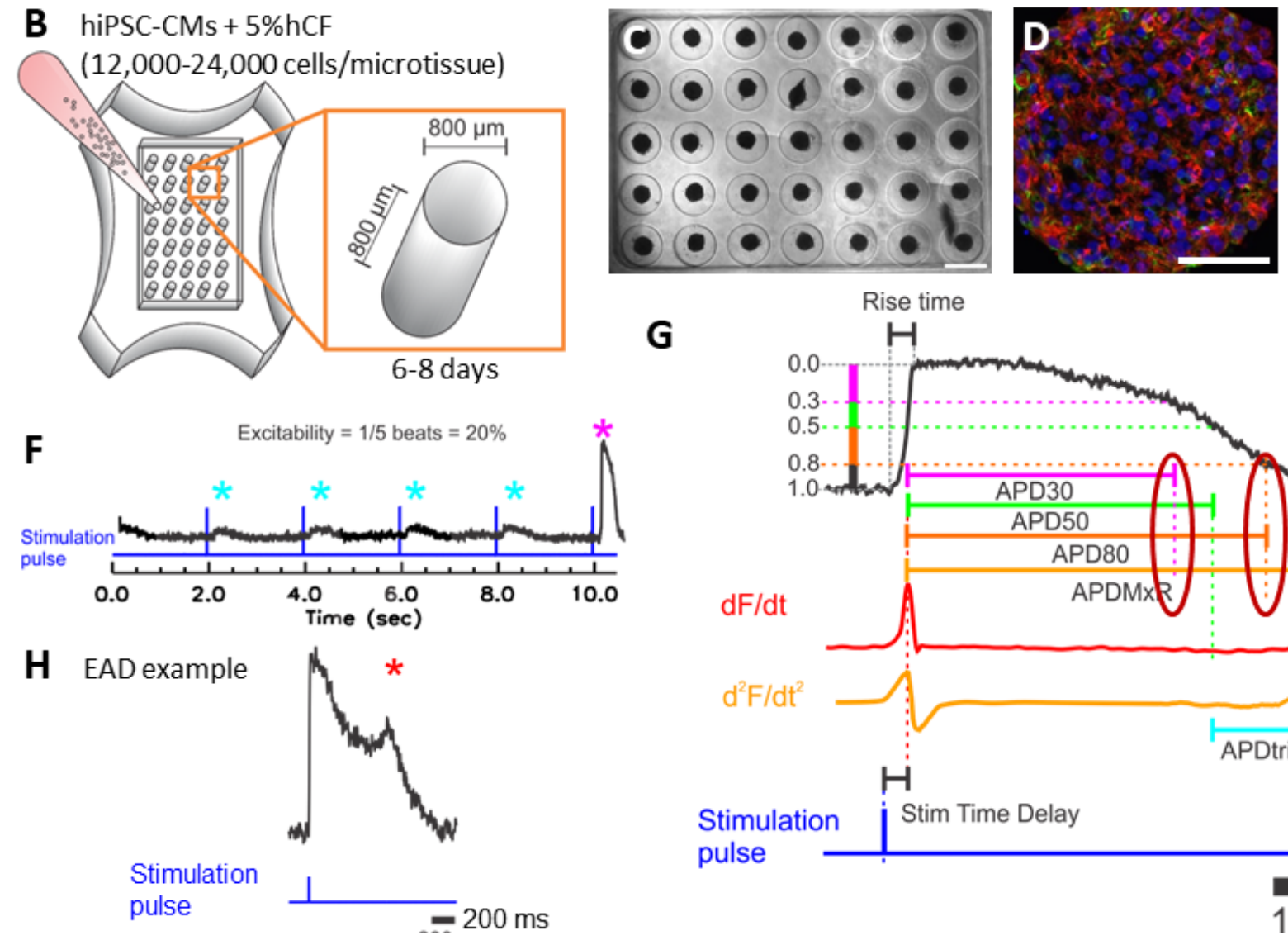

G

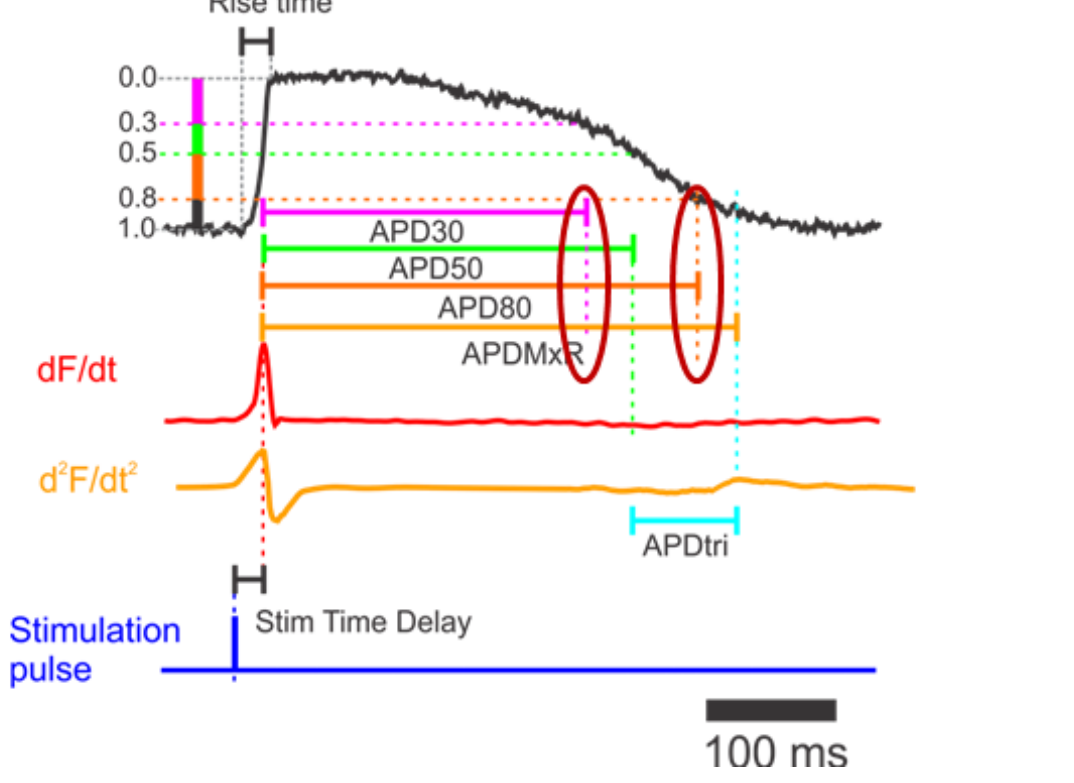

\section{Figure 1}

Differentiation of hiPSC-CM, formation of cardiac microtissues, and definitions of metrics. (A) Timeline shows cardiomyocyte differentiation from human-induced pluripotent stem cells (hiPSCs) in high density 2D culture. Cardiac directed differentiation was achieved with Wnt activation at day 1 and inhibition at day 3 (see Methods). Cardiac phenotype, visually confirmed by beating cells, appeared between days 8 and 12. Cardiomyocytes differentiated from hiPSCs were used for the production of microtissues or were further purified with a lactate-based metabolic selection protocol. Microtissues self-assembled in microwells after 14-28 days of differentiation of hiPSC-CMs (i.e., without or with lactate purification) and addition of human cardiac fibroblasts (hCFs), and they were cultured in the presence of $1 \mathrm{~Hz}$ electrical field stimulation (estim). (B) Schematic of three-dimensional (3D) cardiac microtissue generation shows non-adhesive agarose gels with cylindrical recesses with hemispherical bottoms that guide selfassembly. Cardiac microtissues were cultured for 6-8 days with $1 \mathrm{~Hz}$ pacing. (C) Phase contrast image shows consistent spherical microtissue formation after 5 days of 3D culture in all 35 microwells. Scale bar, $800 \mu \mathrm{m}$. (D) Confocal image shows a representative cardiac troponin I (red), vimentin (green), and DAPI stained cryosection (10 $\mu \mathrm{m}$ thick) of a microtissue fixed after 7 days in 3D culture. Scale bar, $50 \mu \mathrm{m}$. 
(E) Fluorescence image of microtissues at 3.2x magnification was obtained during optical mapping.

Typically, the action potentials (APs) from 4-9 microtissues were recorded simultaneously. (F-H)

Schematics of the AP metrics of that were defined (with units) as: (F) "excitability" (\%) measured from the percentage of captured APs during $10 \mathrm{~s}$ duration of recording with $2 \mathrm{sec}$ pacing cycle length, (G) "stimulation time delay" (ms; stim delay) between stimulation pulse and evoked AP upstroke (dF/dtmax), "rise time" (ms) of AP, "AP duration" (ms) to $30 \%, 50 \%$, and $80 \%$ repolarization (APD30, APD50, APD80), "APD to the maximum repolarization rate" (ms; APDMxR) defined as time between AP upstroke and the end of rapid repolarization marked by d2F/dt2max, "APD triangulation" (ms; APDtri) defined as APDMxR - APD50, and (H) occurrence of "early afterdepolarization" (EAD) reported as (\%) of microtissues showing EADs.
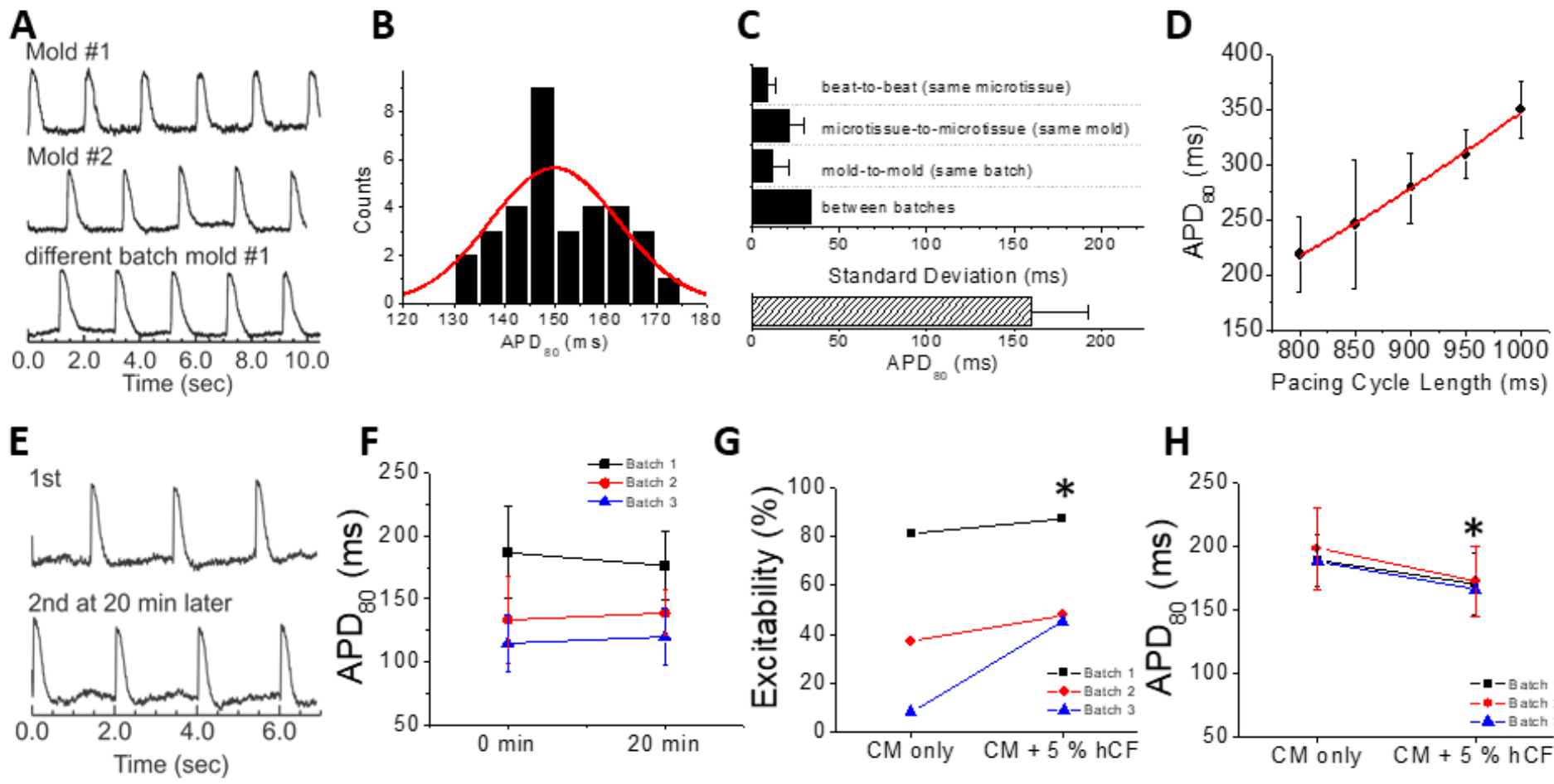

G
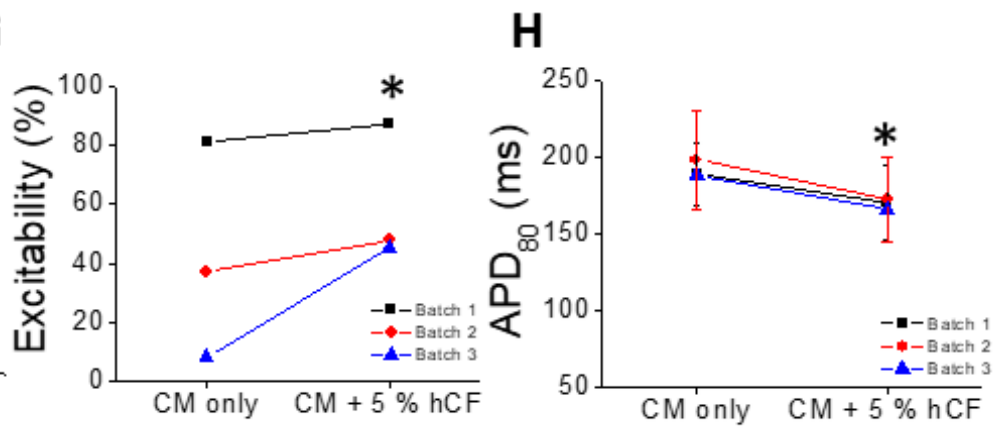

\section{Figure 2}

APD variation and stability from hiPSC-CM microtissues in the absence and presence of $5 \% \mathrm{hCFs}$. HiPSCCMs (without lactate purification) were seeded in microwells without or with $5 \% \mathrm{hCFs}$. After 6-8 days in 3D culture, microtissues were loaded with di-4-ANEPPS for optical mapping of membrane potentials $(\mathrm{Vm})$ under paced conditions. (A) Representative Vm traces from individual microtissues in different molds 
(biological replicates) and different batches (experimental replicates). Values were averaged from all pixels of single microtissues. Only microtissues greater than 60 pixels were analyzed (typically 170 pixels/microtissue). (B) APD80 distribution from 35 microtissues in a single mold is normally distributed. (C) APD variation was assessed for technical, biological, and experimental replicates for APDs measured at $0.5 \mathrm{~Hz}$. The standard deviation from beat-to-beat in the same microtissue (technical replicates) was 8.7 $\pm 4.8 \mathrm{~ms}$ ( $\mathrm{n}>70$ microtissues per batch, $\mathrm{n}=7$ batches), from microtissue-to-microtissue in the same mold (biological replicates) was $20.9 \pm 8.4$ ( $n=2-3$ molds per batch, $n=7$ batches), from mold-to-mold in the same batch (also biological variability) was $11.7 \pm 8.9 \mathrm{~ms}$ (notably less than microtissue-to-microtissue variability; $\mathrm{n}=3$ molds per batch, $\mathrm{n}=7$ batches), and from batch-to-batch of differentiated hiPSC-CMs (experimental variability and another level of biological variability) was $33.1 \mathrm{~ms}$ ( $\mathrm{n}=7$ batches). Note that the standard deviation reported for batch-to-batch variability does not itself have variation (and therefore no error bar is appropriate). (D) The APD restitution curve displays typical rate-dependent increases in APD 80 with increasing pacing cycle length $(800 \mathrm{~ms}=1.25 \mathrm{~Hz}=75 \mathrm{bpm}$ and $1000 \mathrm{~ms}=1 \mathrm{~Hz}=60 \mathrm{bpm})$. (E) Representative $V m$ traces recorded from the same microtissue at 2 time points 20 min apart show consistency of AP during optical mapping. (F) Quantification of the stability of APD80 over 20 min for 3 batches of microtissues (batch \#1: $185.2 \pm 41.6$ vs. $184.0 \pm 29.3, p=0.84$; batch \#2: $114.7 \pm 22.2$ vs. $176.4 \pm 27.1, p=0.46$; batch \#3: $138.6 \pm 17.8$ vs. $119.9 \pm 22.7, p=0.36)$. $(G, H)$ Comparison of excitability $(\mathrm{G})$ and APD80 $(\mathrm{H})$ from microtissues within the same batch of hiPSC-CMs without and with $5 \% \mathrm{hCF}(\mathrm{n}=$ 3 hiPSC-CM batches). Values are means \pm SD. ${ }^{*} P<0.05$ (G, Fisher exact test; $H$, paired t-test). 
A

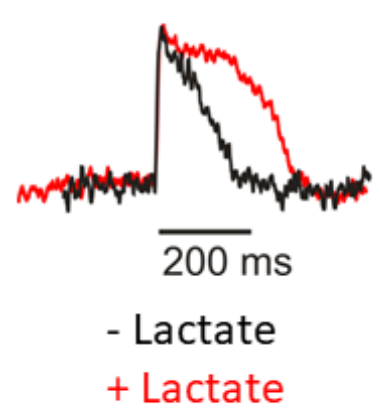

D

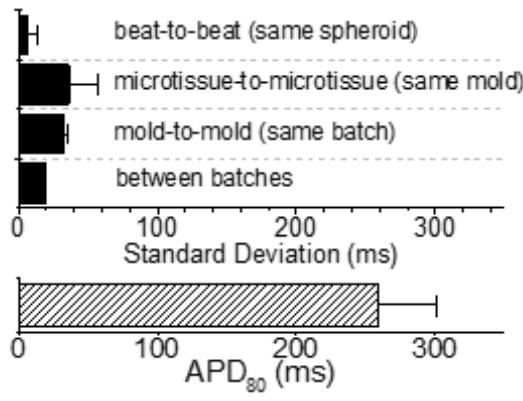

B

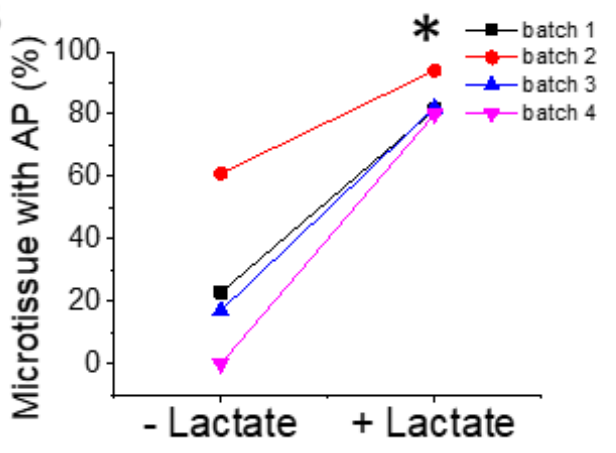

$\mathbf{E}$

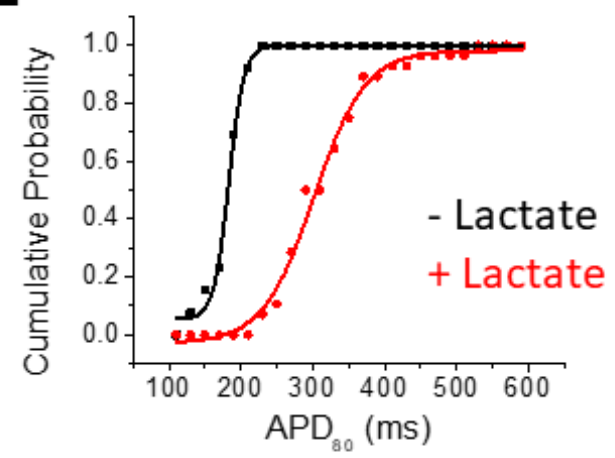

C

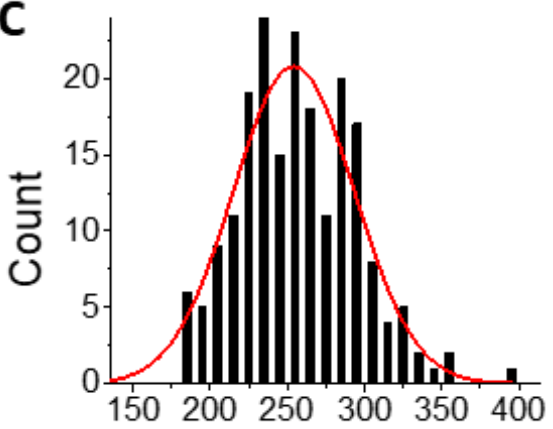

$\mathrm{APD}_{80}(\mathrm{~ms})$

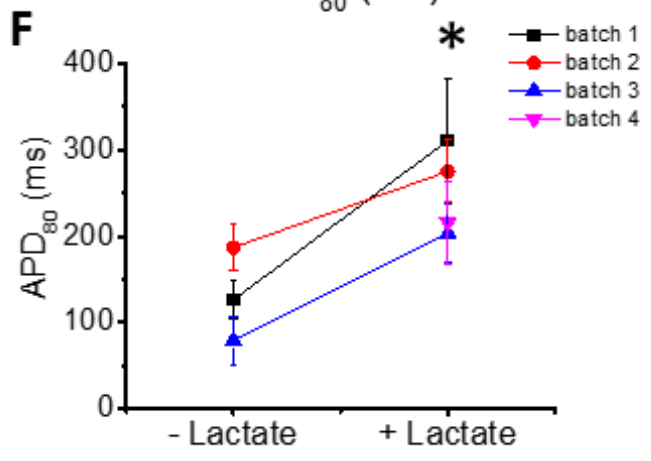

\section{Figure 3}

Lactate purification of hiPSC-CMs prior to microtissue formation with $5 \%$ hCFs improves excitability and lengthens APD. (A) Representative Vm traces from microtissues formed from hiPSC-CMs with (red) and without lactate purification (black) plus $5 \% \mathrm{hCFs}$. HiPSC-CMs from the same differentiation batch but grown in 2D culture without the lactate selection protocol for 28 days (to match the age of the cells) were used as the most appropriate control for comparison. (B) Percent of hiPSC-CM microtissues showing APs during $0.5 \mathrm{~Hz}$ pacing increases with lactate purification of hiPSC-CMs ( $\mathrm{n}=4$ batches, minimum 2 molds per batch). (C) APD80 distribution from 35 microtissues in a single mold with hiPSC-CMLP and $5 \% \mathrm{hCF}$. (D) The average APD80 was $259.0 \pm 42.0 \mathrm{~ms}(n=201)$. APD80 variation between batches is small with hiPSC-CMLP: APDs were measured at $0.5 \mathrm{~Hz}$ cycle length pacing. The standard deviation from beat-tobeat in the same microtissue (technical replicates) was $6.4 \pm 6.5 \mathrm{~ms}(\mathrm{n}>35$ microtissues per batch, $\mathrm{n}=4$ batches), from microtissue-to-microtissue in the same mold (biological replicates) was $36.1 \pm 21.4 \mathrm{~ms}$ ( $\mathrm{n}$ = 2-3 molds per batch, $\mathrm{n}=4$ batches), from mold-to-mold in the same batch (also biological variability) was $31.8 \pm 3.8 \mathrm{~ms}$ ( $n=2-3$ molds per batch, $n=4$ batches), and from batch-to-batch, the standard deviation (another level of biological variability) was $19.3 \mathrm{~ms}(\mathrm{n}=4$ batches). (E) Cumulative probability 
plot for APD80 ( $n=3-4$ batches, at least 2 molds per batch) shows consistently longer APD80 with hiPSCCMLP (red) vs. hiPSC-CM (black). (F) Comparison of APDs from microtissues from the same experimental batch (each color) with and without lactate purification ( $\mathrm{n}=4$ batches, $\geq 2$ molds per batch). Values are means $\pm S D$. $\left.{ }^{*}<0.05\right)$. Note that in batch 4 , the microtissues generated from hiPSC$\mathrm{CM}$ without lactate purification were not excitable.
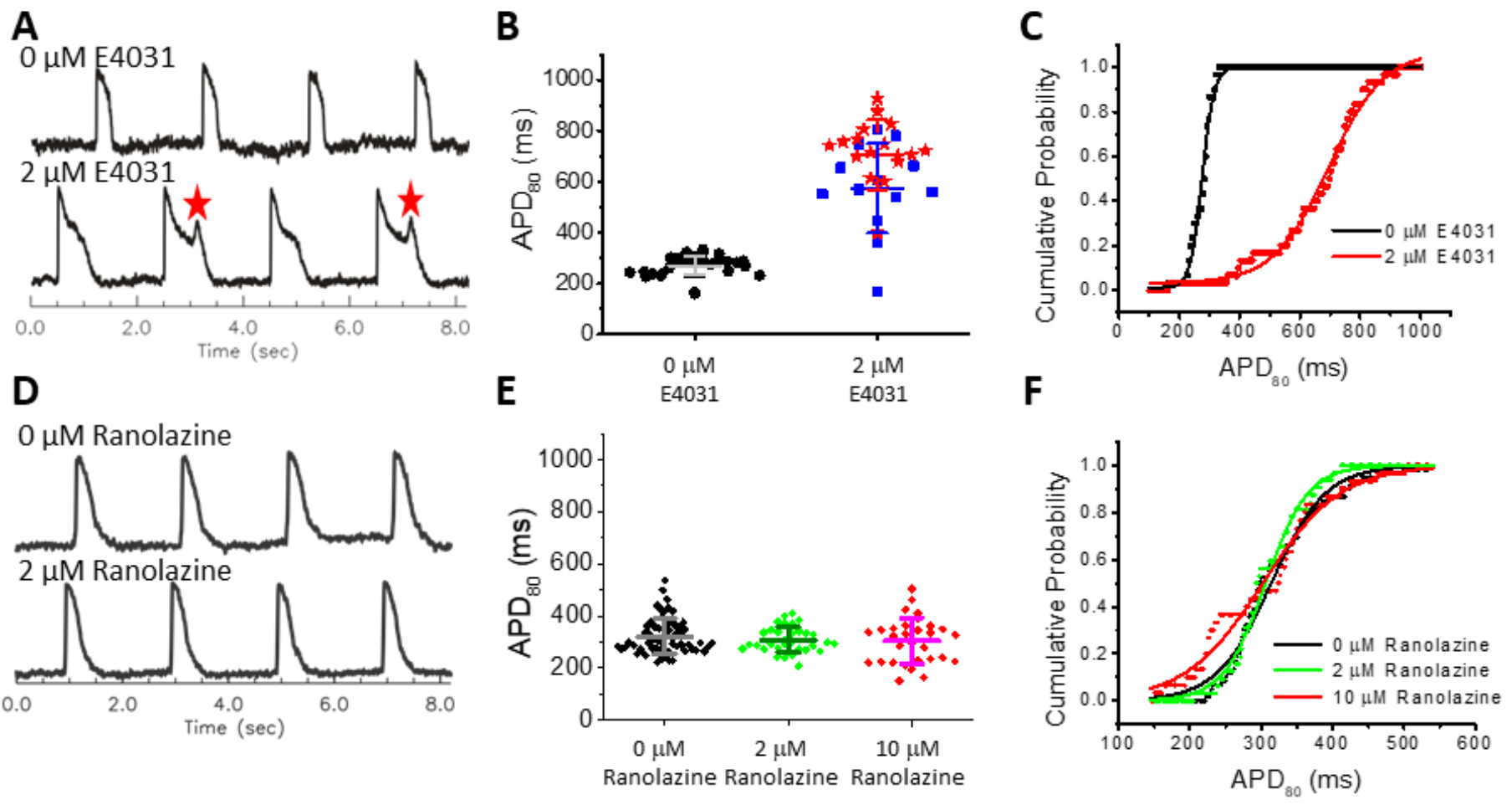

\section{Figure 4}

Differentiation between compounds with high risk (E4301) and low risk (ranolazine) for arrhythmia in hiPSC-CMLP with $5 \%$ hCF microtissues. (A) Representative Vm traces from a microtissue (exposed to DMSO vehicle) before and after exposure to E4031 ( $0 \mu \mathrm{M}$ and $2 \mu \mathrm{M}$, respectively). EADs are marked with red stars. (B, C) Scatter plot (B) and cumulative probability (C) of APD80 ( $n=33$ microtissues per group). Values shown by lines in (B) are means $\pm S D$, and APs with EADs are displayed with red stars. $0 \mu \mathrm{M}$ control was significantly different from each group (vs. no EAD-blue and vs. EAD-red, $p<0.05$ ). (D) Representative Vm traces from a microtissue before and after exposure to a therapeutic dose $(2 \mu \mathrm{M})$ of ranolazine. $(E, F)$ Scatter plot $(E)$ and cumulative probability plot $(F)$ for APD80 $(n=31)$. Values shown by lines in $(E)$ are means \pm SD. APD was not significantly different between any doses of ranolazine. 
A
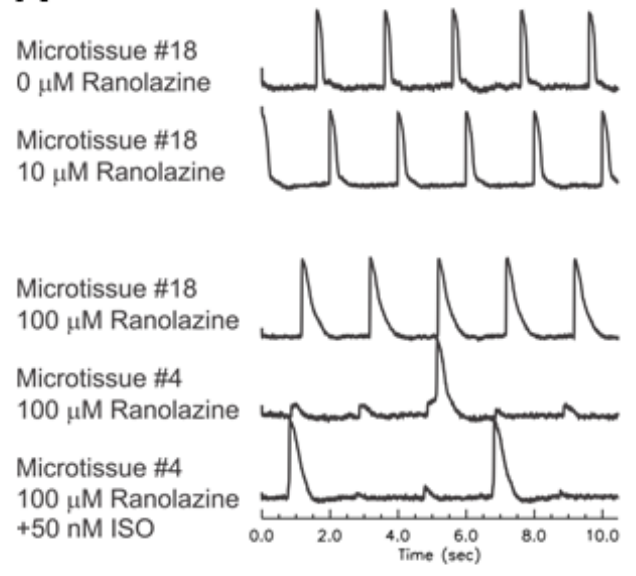

D

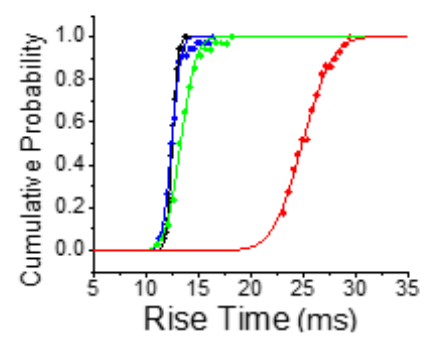

B

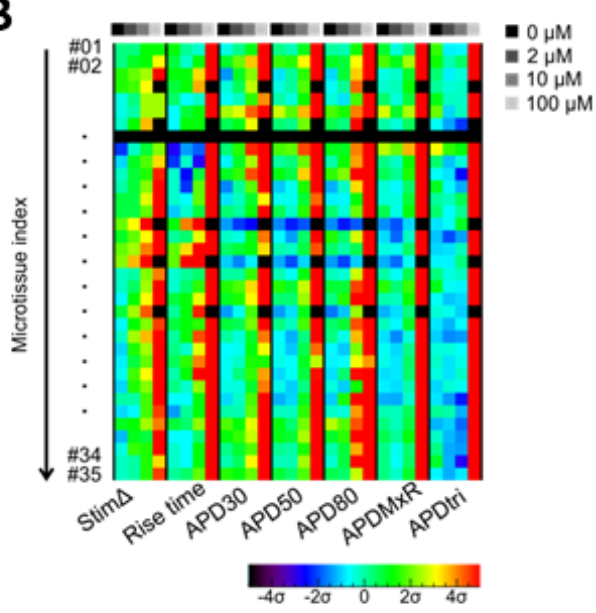

$\mathbf{F}$

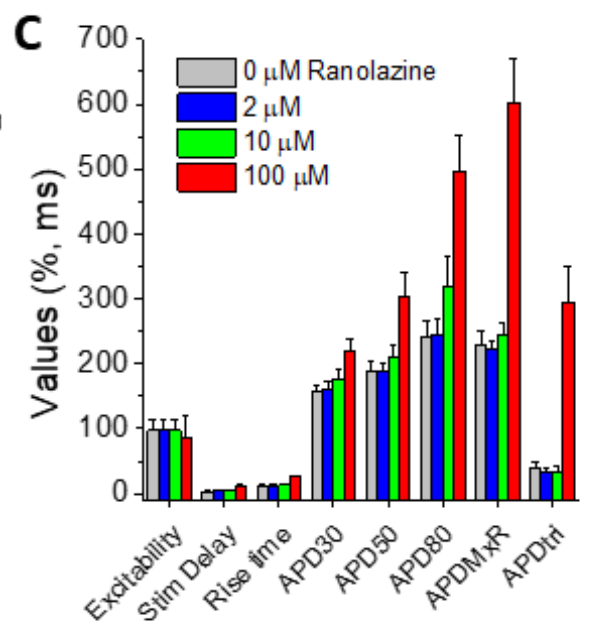

G $\quad-0 \mu \mathrm{M} \operatorname{Ran} \cdot-10 \mu \mathrm{M} \operatorname{Ran}$

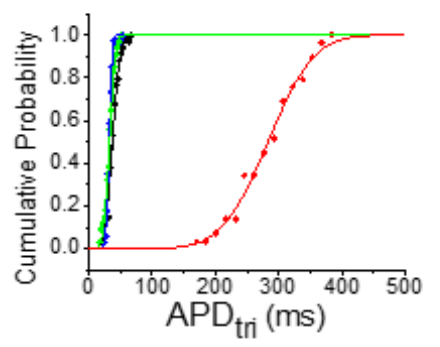

\section{Figure 5}

Dose-dependent effects of ranolazine on AP manifested at high concentrations above the therapeutic window in hiPSC-CMLP with 5\% hCF microtissues. (A) Representative Vm traces from 0, 10, $100 \mu \mathrm{M}$ ranolazine, and $100 \mu \mathrm{M}$ ranolazine plus $50 \mathrm{nM}$ ISO show AP stability at $10 \mu \mathrm{M}$ and instability in microtissues exposed to $100 \mu \mathrm{M}$ Ran with or without ISO . (B) AP metric color map shows each microtissue (rows) and seven AP metrics (major columns) with dose-dependent response at indicated concentrations (minor columns). The color displayed shows shifts as measured by the standard deviation $(\sigma)$ from the average value of the AP metric under the control condition $(0 \mu \mathrm{M})$. Increases appear in the warm color spectrum (progressing yellow-orange-red) and decreases appear in the cool color spectrum (progressing cyan-blue-purple). Black indicates non-excitable microtissues. When excitability is lost in a microtissue, other metrics could not be measured. (C) A summary bar graph for all eight AP metrics shows dose-dependent changes. Values are means \pm SD $(n=35)$. See Supplemental Table 1 (top) for $p$ values. (D-G) Cumulative probability plots for rise time (D), APD80 (E), APDMxR (F), and APDtri (G) show relative stability up to $10 \mu \mathrm{M}$ and marked changes and increased variability (shown by the reduced slope of the curve) at $100 \mu \mathrm{M}$ ranolazine. 


\section{A}

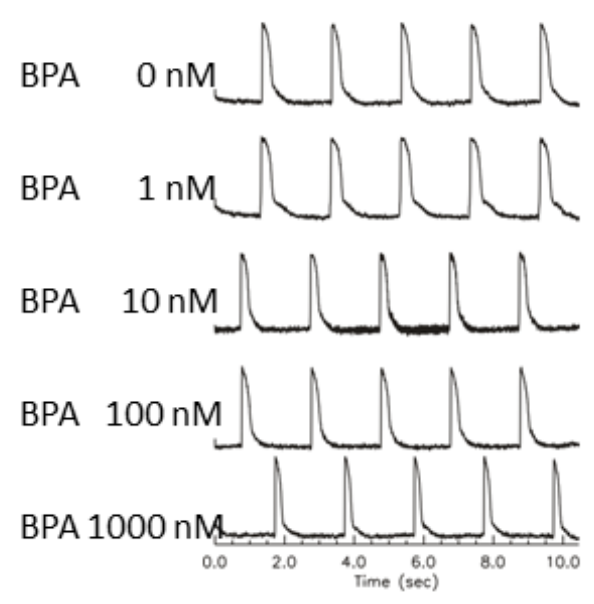

D

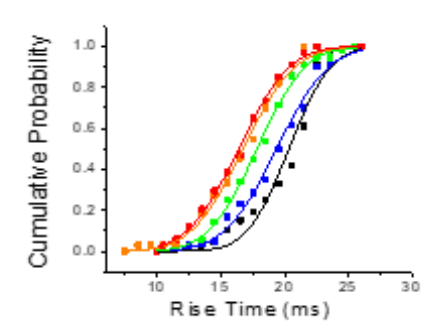

B

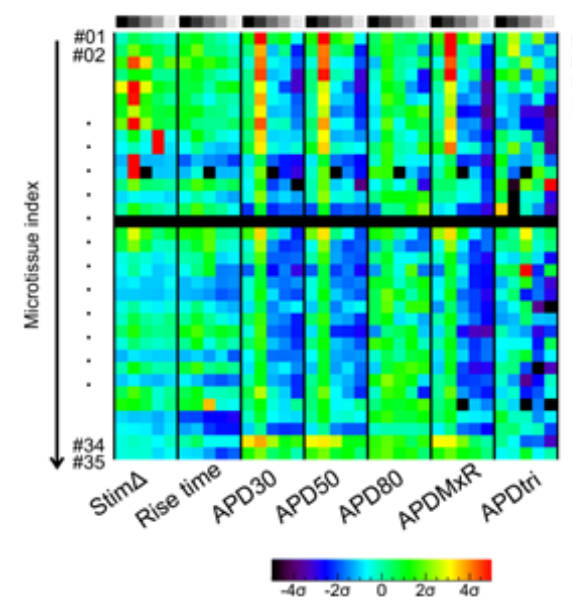

$\mathbf{E}$
$\mathbf{F}$
C

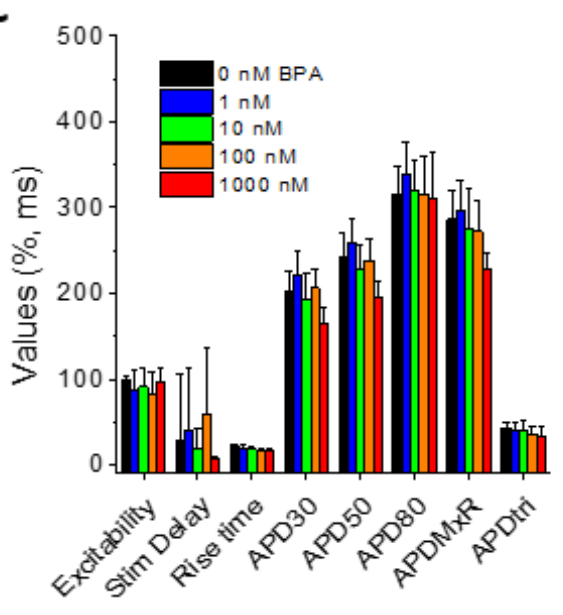

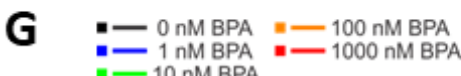

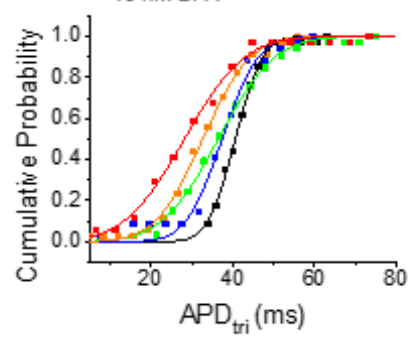

\section{Figure 6}

AP changes in response to the environmental chemical BPA in hiPSC-CMLP with $5 \%$ hCF microtissues. (A) Representative Vm traces from 0,1,10,100, and $1000 \mathrm{nM}$ BPA stimulated at $0.5 \mathrm{~Hz}$. (B) AP metric color map shows a trend of APD shortening (blue colors) at higher doses and a minority of microtissues having a biphasic effect with increases (red-orange) in stim $\triangle$ and APD metrics at $1 \mathrm{nM}$. Key to the panel is as described in Figure 5B. (C) AP parameter summary bar graph. Values are means \pm SD. See Supplemental Table 1 (bottom) for $p$ values. (D-G) Cumulative probability plots for rise time (D), APD80 $(E), \operatorname{APDMxR}(\mathrm{F})$, and APDtri (G). 

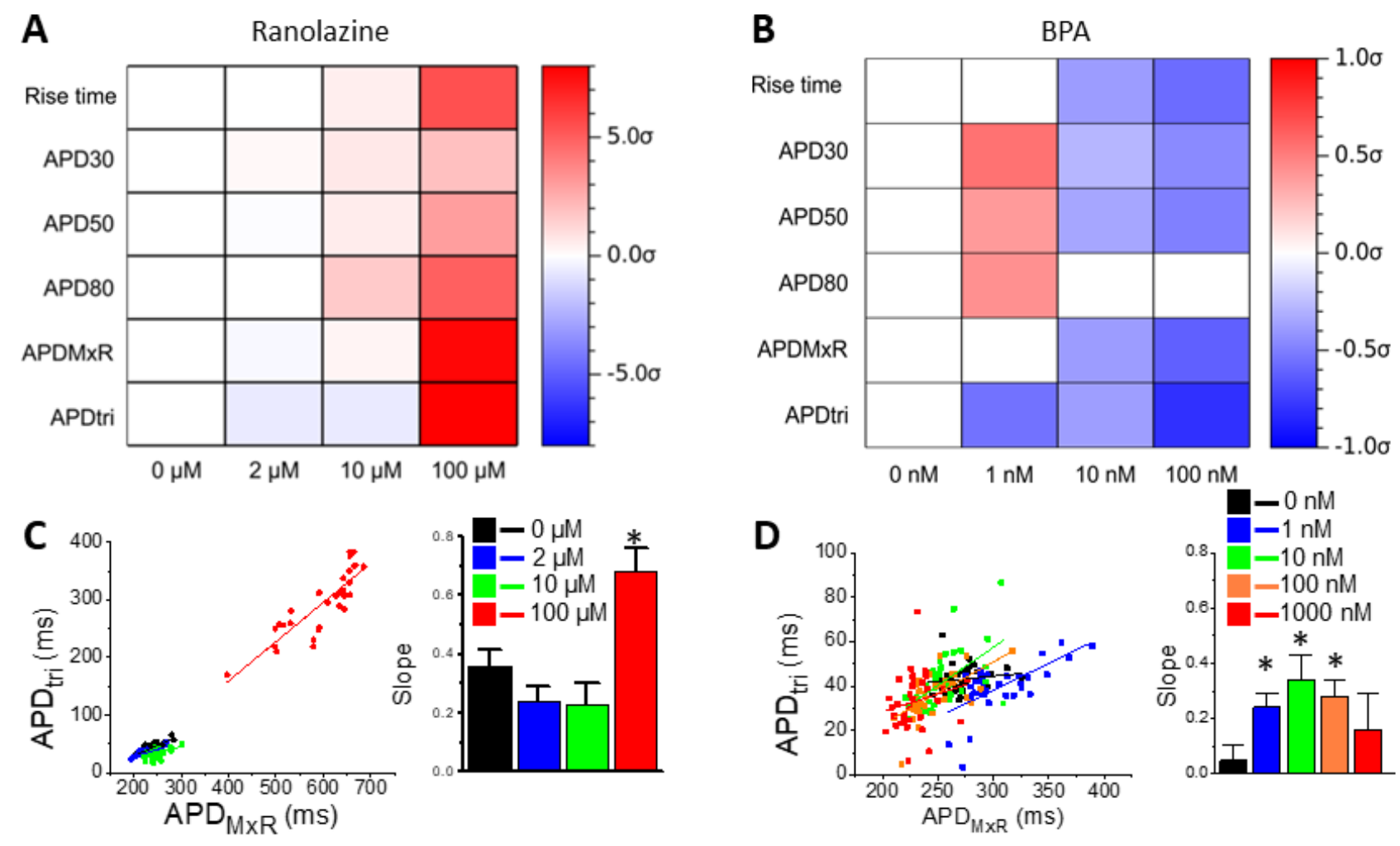

\section{Figure 7}

Characteristic AP metric changes induced by Ranolazine and BPA. The color maps of mean difference AP metrics by ranolazine (A) and BPA (B). The mean differences of AP metrics were normalized by each metric's standard deviation in control ( $\sigma \mathrm{CTR})$. Statistically significant increase and decrease $(p<0.05$, paired t-test) are presented by red and blue color, respectively. (C and D) APDtri versus APD $\neg$ MxR plots in response to increasing concentrations of ranolazine $(C)$ and BPA $\left(D ;{ }^{*} p<0.01\right)$.

\section{Supplementary Files}

This is a list of supplementary files associated with this preprint. Click to download.

- CardioToxSupplementalFigures.docx

- MovieS1GiPSCCMspheroid.mov

- MovieS2normal.wmv

- Movies3EAD.wmv 